\title{
A MATRIX PENCIL APPROACH TO THE ROW BY ROW DECOUPLING PROBLEM FOR DESCRIPTOR SYSTEMS*
}

\author{
DELIN $\mathrm{CHU}^{\dagger}$ AND Y. S. HUNG
}

\begin{abstract}
The row by row decoupling problem (RRDP) for descriptor systems is considered using proportional state feedback and input transformation. Necessary and sufficient conditions for the solvability of the RRDP are provided. These solvability conditions can be readily verified. A constructive solution to the RRDP is given so that the desired feedback and input transformation matrices can be obtained by a numerically reliable procedure.
\end{abstract}

Key words. row by row decoupling, descriptor systems, orthogonal transformation

AMS subject classifications. 93B05, 93B40, 93B52, 65F35

DOI. $10.1137 / \mathrm{S} 089547980343905 \mathrm{X}$

1. Introduction. The row by row decoupling problem (RRDP) has played a central role in classical as well as modern control theory, since it provides a powerful methodology to reduce a multi-input/multioutput complex system to a set of single input/single output systems, thus facilitating a decoupled control strategy of such systems. The row by row decoupling is usually required for ease of system operations, for example, in the process and chemical industries $[1,2]$.

Consider descriptor systems of the form

$$
\left\{\begin{array}{l}
E \dot{x}=A x+B u \\
y=C x
\end{array}\right.
$$

where $E, A \in \mathbf{R}^{n \times n}, B \in \mathbf{R}^{n \times m}, C \in \mathbf{R}^{m \times n}, E$ is singular, $x \in \mathbf{R}^{n}$ is the state, $u \in \mathbf{R}^{m}$ is the control input, and $y \in \mathbf{R}^{m}$ is the output. It is well known that the existence and uniqueness of (classical) solutions to (1) are guaranteed if $(E, A)$ is regular, i.e., $\operatorname{det}(\alpha E-\beta A) \neq 0$ for some $(\alpha, \beta) \in \mathbf{C}^{2}$. The system (1) is said to have index at most one if the dimension of the largest nilpotent block in the Kronecker canonical form of $(E, A)$ is at most one [17].

Descriptor systems that are regular and of index at most one can be separated into purely dynamical and purely algebraic parts (fast and slow modes). If the index is larger than 1, then impulses can arise in the response of the system if the control is not sufficiently smooth $[7,17]$. Therefore, in the design of feedback control, one should ensure that the closed-loop system is regular and of index at most one.

If we apply state feedback of the form

$$
u=F x+H v
$$

to the descriptor system (1), then the closed-loop system becomes

$$
\left\{\begin{array}{l}
E \dot{x}=(A+B F) x+B H v \\
y=C x
\end{array}\right.
$$

* Received by the editors December 23, 2003; accepted for publication (in revised form) by B. T. Kågström March 7, 2006; published electronically September 19, 2006.

http://www.siam.org/journals/simax/28-3/43905.html

${ }^{\dagger}$ Department of Mathematics, National University of Singapore, 2 Science Drive 2, Singapore 117543 (matchudl@math.nus.edu.sg).

${ }_{\ddagger}^{\ddagger}$ Department of Electrical and Electronic Engineering, University of Hong Kong, Pokfulam Road, Hong Kong (yshung@hkueee.hku.hk). The work of this author was supported by a CRCG research grant from the University of Hong Kong. 
The problem to be considered can be stated as follows.

The RRDP. Given a descriptor system of the form (1), determine a state feedback matrix $F \in \mathbf{R}^{m \times n}$ and a nonsingular input transformation matrix $H \in \mathbf{R}^{m \times m}$ such that

(a) the pencil $(E, A+B F)$ is regular and of index at most one;

(b) the closed-loop transfer function matrix

$$
C(s E-A-B F)^{-1} B H \quad \text { is nonsingular and diagonal. }
$$

Here $C(s E-A-B F)^{-1} B H$ being nonsingular means that

$$
\operatorname{rank}\left(C(s E-A-B F)^{-1} B H\right)=m \quad \text { for some } s \in \mathbf{C} .
$$

Before studying the RRDP for the descriptor system (1), we summarize the main results on the RRDP available in the literature.

The RRDP for linear time-invariant systems of the form

$$
\left\{\begin{array}{l}
\mathcal{E} \dot{x}=\mathcal{A} x+\mathcal{B} u \\
y=\mathcal{C} x
\end{array}\right.
$$

with $\mathcal{E}, \mathcal{A} \in \mathbf{R}^{k \times k}, \mathcal{B} \in \mathbf{R}^{k \times p}, \mathcal{C} \in \mathbf{R}^{p \times k}$, and $\mathcal{E}$ nonsingular has been investigated extensively over the last three decades and is still attracting continuing interests $[2,3,4,5,6,9,10,21]$. In particular, because system (5) is equivalent to

$$
\left\{\begin{array}{l}
\dot{x}=\mathcal{E}^{-1} \mathcal{A} x+\mathcal{E}^{-1} \mathcal{B} u \\
y=\mathcal{C} x
\end{array}\right.
$$

we have the following theorem.

TheOREM 1 (see [3]). Given system (5) with $\mathcal{E}$ nonsingular, let $c_{i}$ be the ith row of $\mathcal{C}$. If

$$
c_{i}\left(\mathcal{E}^{-1} \mathcal{A}\right)^{j}\left(\mathcal{E}^{-1} \mathcal{B}\right) \neq 0
$$

for some nonnegative integer $j$, then set

$$
l_{i}=\min \left\{j \geq 0: j \text { is integer satisfying } c_{i}\left(\mathcal{E}^{-1} \mathcal{A}\right)^{j}\left(\mathcal{E}^{-1} \mathcal{B}\right) \neq 0\right\} ;
$$

otherwise, set $l_{i}=k-1$. Define

$$
\mathcal{L}=\left[\begin{array}{c}
c_{1}\left(\mathcal{E}^{-1} \mathcal{A}\right)^{l_{1}} \\
c_{2}\left(\mathcal{E}^{-1} \mathcal{A}\right)^{l_{2}} \\
\vdots \\
c_{p}\left(\mathcal{E}^{-1} \mathcal{A}\right)^{l_{p}}
\end{array}\right]\left(\mathcal{E}^{-1} \mathcal{B}\right), \quad \mathcal{K}=\left[\begin{array}{c}
c_{1}\left(\mathcal{E}^{-1} \mathcal{A}\right)^{l_{1}+1} \\
c_{2}\left(\mathcal{E}^{-1} \mathcal{A}\right)^{l_{2}+1} \\
\vdots \\
c_{p}\left(\mathcal{E}^{-1} \mathcal{A}\right)^{l_{p}+1}
\end{array}\right]\left(\mathcal{E}^{-1} \mathcal{B}\right)
$$

Then the RRDP for system (5) is solvable if and only if the matrix $\mathcal{L}$ is nonsingular. In this case, a solution pair $(\mathcal{F}, \mathcal{H})$ is given by

$$
\mathcal{F}=-\mathcal{L}^{-1} \mathcal{K}, \quad \mathcal{H}=\mathcal{L}^{-1} .
$$

Although Theorem 1 provides an explicit solution for the RRDP of the linear time-invariant system (5) with $\mathcal{E}$ nonsingular, some natural questions remain.

(a) If the RRDP is solvable,

(i) can we solve the RRDP with the additional requirement of stability?

(ii) does numerically reliable solution exist for the RRDP with stability? 
(b) If the RRDP is not solvable, can one resort to a relaxed problem of triangular decoupling?

Regarding (a)(i), the RRDP with stability for the system (5) has been investigated in $[5,6]$ using geometric and structural approaches, giving coordinate-free solvability conditions. But, the results in $[5,6]$ cannot lead to numerically reliable methods for computing a solution to the problem. To address (a)(ii), a numerically reliable method has been developed in [14] based on orthogonal transformations. If the condition of Theorem 1 is not satisfied so that the RRDP is not solvable, it is shown in [15] that a triangular decoupling problem may be solvable under less restrictive conditions. In [15], explicit solvability conditions are provided with a parameterization of all solutions to the triangular decoupling problem.

Unfortunately, the above results for the RRDP for the system (5) with $\mathcal{E}$ nonsingular cannot be readily extended to the general descriptor system (1). For example, it is not possible to apply existing results to system (1) by decomposing it into differential and algebraic parts and then deal with them separately. Instead, it is necessary to develop a separate theory to handle the RRDP for descriptor systems. The RRDP for descriptor system (1) has been studied in $[7,11,19]$. In [7], it is shown that the RRDP for system (1) is solvable using combined proportional and derivative state feedback if and only if the input-output transfer function is invertible. However, the use of derivative feedback is undesirable due to noise accentuation and an increase in the system order. To our knowledge, the solution is still not known for the RRDP of the descriptor system (1) using only proportional state feedback.

A problem related to the RRDP is the disturbance decoupling problem. Although the objectives of the RRDP are different from the disturbance decoupling problem, we will make use of the matrix pencil approach developed in $[12,13]$ to characterize necessary and sufficient conditions for the solvability of the RRDP for the system (1). In this paper, we provide numerically reliable methods for verifying the solvability of the RRDP for the system (1) and for computing the solution matrices $F$ and $H$. These results are new to our knowledge and are valuable, as real descriptor systems with singular $E$ do exist in practice. However, the RRDP with stability for the descriptor system (1) remains an open problem.

The paper is organized as follows. Some necessary preliminary results for matrix pencils are collected in section 2. In section 3, necessary and sufficient solvability conditions as well as a numerically reliable algorithm for the RRDP of descriptor system (1) are established. Concluding remarks are included in section 4.

2. Preliminaries. The following two lemmas are basic results for matrix pencils and will be needed in the development to be given in the next section.

Lemma 2 (see $[12,14]$ ). Given $\mathcal{E}, \mathcal{A} \in \mathbf{R}^{n \times n}, \mathcal{B} \in \mathbf{R}^{n \times m}, \mathcal{C} \in \mathbf{R}^{p \times n}$, and $\mathcal{D} \in$ $\mathbf{R}^{p \times m}$ with $\mathcal{E}$ nonsingular,

(i)

$$
\mathcal{C}(s \mathcal{E}-\mathcal{A})^{-1} \mathcal{B}+\mathcal{D}=0
$$

if and only if

$$
\mathcal{D}=0, \quad \max _{s \in \mathbf{C}} \operatorname{rank}\left[\begin{array}{cc}
s \mathcal{E}-\mathcal{A} & \mathcal{B} \\
\mathcal{C} & 0
\end{array}\right]=n
$$

(ii) assume

$$
\operatorname{rank}\left[\begin{array}{cc}
s \mathcal{E}-\mathcal{A} & \mathcal{B}
\end{array}\right]=n \quad \forall s \in \mathbf{C} .
$$


Then

$$
\max _{s \in \mathbf{C}} \operatorname{rank}\left[\begin{array}{cc}
s \mathcal{E}-\mathcal{A} & \mathcal{B} \\
\mathcal{C} & -\mathcal{D}
\end{array}\right]=n
$$

if and only if

$$
\mathcal{C}=0, \quad \mathcal{D}=0
$$

Lemma 3. Given $\mathcal{E}, \mathcal{A} \in \mathbf{R}^{n \times l}, \mathcal{B} \in \mathbf{R}^{n \times m}, \mathcal{C} \in \mathbf{R}^{p \times n}$, and $\mathcal{D} \in \mathbf{R}^{p \times m}$,

(i)

$$
\max _{s \in \mathbf{C}} \operatorname{rank}\left[\begin{array}{cc}
s \mathcal{E}-\mathcal{A} & \mathcal{B} \\
\mathcal{C} & -\mathcal{D}
\end{array}\right] \geq \operatorname{rank}(\mathcal{E})+\operatorname{rank}(\mathcal{D})
$$

(ii) if $\mathcal{E}$ and $\mathcal{D}$ are of full row rank, then

$$
\max _{s \in \mathbf{C}} \operatorname{rank}\left[\begin{array}{cc}
s \mathcal{E}-\mathcal{A} & \mathcal{B} \\
\mathcal{C} & -\mathcal{D}
\end{array}\right]=n+p .
$$

Proof. Part (i). We can assume without loss of generality that

$$
\left.\mathcal{D}=\left[\begin{array}{cc}
\mathcal{D}_{11} & 0 \\
0 & 0
\end{array}\right]\right\} p-\tau
$$

with $\mathcal{D}_{11}$ nonsingular and $\operatorname{rank}(\mathcal{D})=\operatorname{rank}\left(\mathcal{D}_{11}\right)=\tau$. Denote

$$
\left.\mathcal{B}=\left[\begin{array}{cc}
\tau & m-\tau \\
\mathcal{B}_{1} & \mathcal{B}_{2}
\end{array}\right], \quad \mathcal{C}=\left[\begin{array}{l}
\mathcal{C}_{1} \\
\mathcal{C}_{2}
\end{array}\right]\right\} \begin{aligned}
& \} \\
& \} p-\tau
\end{aligned},
$$

and let the generalized upper triangular form $[8,20]$ of

$$
\left[\begin{array}{cc}
s \mathcal{E}-\mathcal{A}+\mathcal{B}_{1} \mathcal{D}_{11}^{-1} \mathcal{C}_{1} & \mathcal{B}_{2} \\
\mathcal{C}_{2} & 0
\end{array}\right]
$$

be

$$
\begin{aligned}
& \mathcal{P}\left[\begin{array}{cc}
s \mathcal{E}-\mathcal{A}+\mathcal{B}_{1} \mathcal{D}_{11}^{-1} \mathcal{C}_{1} & \mathcal{B}_{2} \\
\mathcal{C}_{2} & 0
\end{array}\right] \mathcal{Q} \\
& \begin{array}{llll}
\mu_{1} & \mu_{2} & \mu_{3} & \mu_{4}
\end{array} \\
& =\left[\begin{array}{cccc}
s \Theta_{11}-\Phi_{11} & s \Theta_{12}-\Phi_{12} & s \Theta_{13}-\Phi_{13} & s \Theta_{14}-\Phi_{14} \\
0 & s \Theta_{22}-\Phi_{22} & s \Theta_{23}-\Phi_{23} & s \Theta_{24}-\Phi_{24} \\
0 & 0 & s \Theta_{33}-\Phi_{33} & s \Theta_{34}-\Phi_{34} \\
0 & 0 & 0 & s \Theta_{44}-\Phi_{44}
\end{array}\right] \begin{array}{c}
\} \nu_{1} \\
\} \mu_{2} \\
\} \mu_{3} \\
\} \nu_{4}
\end{array},
\end{aligned}
$$

where $\mathcal{P}$ and $\mathcal{Q}$ are orthogonal, $\Theta_{11}$ is of full row rank, $\Theta_{44}$ is of full column rank, $\Theta_{22}$ is nonsingular, and

$$
\operatorname{rank}\left(s \Theta_{11}-\Phi_{11}\right)=\nu_{1}, \quad \operatorname{rank}\left(s \Theta_{33}-\Phi_{33}\right)=\mu_{3}, \quad \operatorname{rank}\left(s \Theta_{44}-\Phi_{44}\right)=\mu_{4} \quad \forall s \in \mathbf{C} .
$$


Now we have that

$\operatorname{rank}(\mathcal{E})=\operatorname{rank}\left[\begin{array}{cccc}\Theta_{11} & \Theta_{12} & \Theta_{13} & \Theta_{14} \\ 0 & \Theta_{22} & \Theta_{23} & \Theta_{24} \\ 0 & 0 & \Theta_{33} & \Theta_{34} \\ 0 & 0 & 0 & \Theta_{44}\end{array}\right]=\nu_{1}+\mu_{2}+\operatorname{rank}\left(\Theta_{33}\right)+\mu_{4} \leq \nu_{1}+\mu_{2}+\mu_{3}+\mu_{4}$

and the equality $\operatorname{rank}(\mathcal{D})=\tau$ yield that

$$
\begin{aligned}
\max _{s \in \mathbf{C}} \operatorname{rank}\left[\begin{array}{ccc}
s \mathcal{E}-\mathcal{A} & \mathcal{B} \\
\mathcal{C} & -\mathcal{D}
\end{array}\right] \\
=\max _{s \in \mathbf{C}} \operatorname{rank}\left[\begin{array}{ccc}
s \mathcal{E}-\mathcal{A} & \mathcal{B}_{1} & \mathcal{B}_{2} \\
\mathcal{C}_{1} & -\mathcal{D}_{11} & 0 \\
\mathcal{C}_{2} & 0 & 0
\end{array}\right] \\
=\max _{s \in \mathbf{C}} \operatorname{rank}\left[\begin{array}{ccc}
s \mathcal{E}-\mathcal{A}+\mathcal{B}_{1} \mathcal{D}_{11}^{-1} \mathcal{C}_{1} & \mathcal{B}_{2} \\
\mathcal{C}_{2} & 0
\end{array}\right]+\tau \\
=\max _{s \in \mathbf{C}} \operatorname{rank}\left[\begin{array}{cccc}
s \Theta_{11}-\Phi_{11} & s \Theta_{12}-\Phi_{12} & s \Theta_{13}-\Phi_{13} & s \Theta_{14}-\Phi_{14} \\
0 & s \Theta_{22}-\Phi_{22} & s \Theta_{23}-\Phi_{23} & s \Theta_{24}-\Phi_{24} \\
0 & 0 & s \Theta_{33}-\Phi_{33} & s \Theta_{34}-\Phi_{34} \\
0 & 0 & 0 & s \Theta_{44}-\Phi_{44}
\end{array}\right]+\tau \\
=\nu_{1}+\mu_{2}+\mu_{3}+\mu_{4}+\tau \geq \operatorname{rank}(\mathcal{E})+\operatorname{rank}(\mathcal{D}) .
\end{aligned}
$$

Part (ii). Since $\mathcal{E}$ and $\mathcal{D}$ are of full row rank, by part (i) we obtain that

$$
\max _{s \in \mathbf{C}} \operatorname{rank}\left[\begin{array}{cc}
s \mathcal{E}-\mathcal{A} & \mathcal{B} \\
\mathcal{C} & -\mathcal{D}
\end{array}\right] \geq \operatorname{rank}(\mathcal{E})+\operatorname{rank}(\mathcal{D})=n+p .
$$

But, it is obvious that

$$
\max _{s \in \mathbf{C}} \operatorname{rank}\left[\begin{array}{cc}
s \mathcal{E}-\mathcal{A} & \mathcal{B} \\
\mathcal{C} & -\mathcal{D}
\end{array}\right] \leq n+p
$$

Hence,

$$
\max _{s \in \mathbf{C}} \operatorname{rank}\left[\begin{array}{cc}
s \mathcal{E}-\mathcal{A} & \mathcal{B} \\
\mathcal{C} & -\mathcal{D}
\end{array}\right]=n+p
$$

The next lemma provides necessary and sufficient conditions for a matrix pencil to be regular and of index at most one.

LEMma 4 (see $[7,17])$. Let $E, A \in \mathbf{R}^{n \times n}$. The following statements are equivalent.

(i) $(E, A)$ is regular and of index at most one.

(ii) $\operatorname{rank}\left[E S_{\infty}(E)\right]=n$, where $S_{\infty}(E)$ denotes a matrix with orthogonal columns spanning the right nullspace of matrix $E$.

(iii) $\operatorname{deg}(\operatorname{det}(s E-A))=\operatorname{rank}(E)$.

3. Main results. The purpose of this section is to present necessary and sufficient solvability conditions as well as a numerically reliable algorithm for the RRDP of descriptor system (1). For this purpose, first we transform the RRDP for descriptor system (1) into the RRDP for a linear time-invariant system using orthogonal transformations. 
THEOREM 5. Given system (1), there exist nonnegative integers $n_{1}, n_{2}, n_{3}, \tilde{n}_{2}$, and $\tilde{n}_{3}$ and orthogonal matrices $U, V, Q \in \mathbf{R}^{n \times n}$, and $W \in \mathbf{R}^{m \times m}$ with

$$
\left.Q=\left[\begin{array}{cc}
n_{1} & n_{2}+n_{3} \\
Q_{11} & Q_{12} \\
Q_{21} & Q_{22}
\end{array}\right]\right\} n_{1} \begin{aligned}
& \\
& \} n_{2}+n_{3}
\end{aligned}
$$

such that $n_{1}+n_{2}+n_{3}=n_{1}+\tilde{n}_{2}+\tilde{n}_{3}=n, Q_{11}$ is nonsingular, and

$$
\begin{aligned}
& {\left[\begin{array}{ccc}
Q_{11} & Q_{12} & 0 \\
0 & I & 0 \\
0 & 0 & I
\end{array}\right]\left[\begin{array}{ll}
U & 0 \\
0 & I
\end{array}\right]\left[\begin{array}{ccc}
s E-A & \mid & B \\
--- & - & -- \\
C & \mid & 0
\end{array}\right]\left[\begin{array}{cc}
V & 0 \\
0 & W
\end{array}\right]} \\
& \begin{array}{ccccc}
n_{1} & n_{2} & n_{3} & \tilde{n}_{2} & m-\tilde{n}_{2}
\end{array}
\end{aligned}
$$

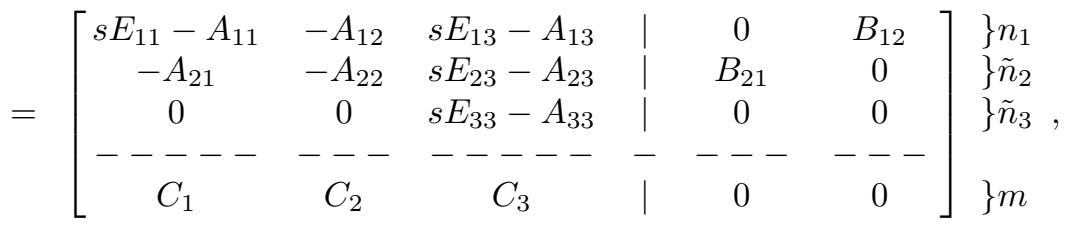

where $E_{11}$ and $B_{21}$ are nonsingular, and $s E_{33}-A_{33}$ is of full column rank for any $s \in \mathbf{C}$.

Proof. The form (9) is constructed in [23].

In the following, we give a system interpretation of the form (9).

With respect to the coordinate transformations in the form (9), the system (1) can be expressed as

$$
\left\{\begin{array}{l}
\left(\left[\begin{array}{cc}
Q_{11} & Q_{12} \\
0 & I
\end{array}\right] U E V\right) V^{T} \dot{x}=\left(\left[\begin{array}{cc}
Q_{11} & Q_{12} \\
0 & I
\end{array}\right] U A V\right) V^{T} x \\
\quad+\left(\left[\begin{array}{cc}
Q_{11} & Q_{12} \\
0 & I
\end{array}\right] U B W\right) W^{T} u, \\
y=(C V) V^{T} x,
\end{array}\right.
$$

where $V^{T} x$ represents the transformed state vector and $W^{T} u$ the transformed input. Let

$$
\left.V^{T} x=\left[\begin{array}{c}
\tilde{x} \\
x_{2} \\
x_{3}
\end{array}\right] \begin{array}{l}
\} n_{1} \\
\} n_{2} \\
\} n_{3}
\end{array}, \quad W^{T} u=\left[\begin{array}{l}
u_{1} \\
u_{2}
\end{array}\right]\right\} \begin{aligned}
& 3 \tilde{n}_{2} \\
& \} m-\tilde{n}_{2}
\end{aligned} .
$$

Then system (10) is equivalent to

$$
\left\{\begin{array}{l}
E_{11} \dot{\tilde{x}}+E_{13} \dot{x}_{3}=A_{11} \tilde{x}+A_{12} x_{2}+A_{13} x_{3}+B_{12} u_{2}, \\
E_{23} \dot{x}_{3}=A_{21} \tilde{x}+A_{22} x_{2}+A_{23} x_{3}+B_{21} u_{1}, \\
E_{33} \dot{x}_{3}=A_{33} x_{3}, \\
y=C_{1} \tilde{x}+C_{2} x_{2}+C_{3} x_{3} .
\end{array}\right.
$$

Because $s E_{33}-A_{33}$ is of full column rank for any $s \in \mathbf{C}$, according to [22], we know that

$$
x_{3}=0 \quad \forall t \geq 0 .
$$

Consequently, $E_{33} \dot{x}_{3}=A_{33} x_{3}$ is a redundant subsystem (associated with $x_{3}$ constrained to be zero). As the redundant subsystem has a zero trajectory $x_{3}=0$, we can delete this part. Therefore, (1) is reduced to 
- a regular subsystem (with nonsingular $E_{11}$ )

$$
\left\{\begin{array}{l}
E_{11} \dot{\tilde{x}}=A_{11} \tilde{x}+\left[\begin{array}{ll}
B_{12} & A_{12}
\end{array}\right]\left[\begin{array}{l}
u_{2} \\
x_{2}
\end{array}\right], \\
y=C_{1} \tilde{x}+\left[\begin{array}{ll}
0 & C_{2}
\end{array}\right]\left[\begin{array}{l}
u_{2} \\
x_{2}
\end{array}\right]
\end{array}\right.
$$

- an algebraic subsystem (associated with $x_{2}$ )

$$
0=A_{21} \tilde{x}+A_{22} x_{2}+B_{21} u_{1} .
$$

The algebraic part of the system results in the algebraic condition (14), which must be satisfied. This can be taken as an algebraic constraint on the feasibility of the system (1). Since $B_{21}$ is nonsingular, we can always find an input $u_{1}$ to ensure that the descriptor system (1) is consistent. If we consider

$$
\left[\begin{array}{l}
u_{2} \\
x_{2}
\end{array}\right]=\tilde{u}
$$

as a new input and choose $u_{1}=-B_{21}^{-1}\left(A_{21} \tilde{x}+A_{22} x_{2}\right)$, then the regular subsystem (13) becomes

$$
\left\{\begin{array}{l}
E_{11} \dot{\tilde{x}}=A_{11} \tilde{x}+\left[\begin{array}{ll}
B_{12} & A_{12}
\end{array}\right] \tilde{u} \\
y=C_{1} \tilde{x}+\left[\begin{array}{ll}
0 & C_{2}
\end{array}\right] \tilde{u}
\end{array}\right.
$$

and the algebraic constraint (14) is satisfied. The regular subsystem (15) preserves the finite zeros of the descriptor system (1), as shown in the next corollary.

Corollary 6. Given a descriptor system (1), let the form (9) be determined. Then the finite zeros of system (1) are the same as those of system (15).

Proof. The finite zeros of systems (1) and (15) are the finite eigenvalues of matrix pencils

$$
\left[\begin{array}{cc}
A-s E & B \\
C & 0
\end{array}\right] \text { and }\left[\begin{array}{ccc}
A_{11}-s E_{11} & B_{12} & A_{12} \\
C_{1} & 0 & C_{2}
\end{array}\right]
$$

respectively. By construction, $B_{21}$ is nonsingular and $s E_{33}-A_{33}$ has full column rank for any $s \in \mathbf{C}$. Hence, we obtain by means of the form (9) that matrix pencils

$$
\left[\begin{array}{cc}
A-s E & B \\
C & 0
\end{array}\right] \text { and }\left[\begin{array}{ccc}
A_{11}-s E_{11} & B_{12} & A_{12} \\
C_{1} & 0 & C_{2}
\end{array}\right]
$$

have the same finite eigenvalues. Therefore, systems (1) and (15) have the same set of finite zeros.

After removing the redundant subsystem $E_{33} \dot{x}_{3}=A_{33} x_{3}$ and assuming that the algebraic consistency (14) is satisfied, it is therefore natural to focus on the regular subsystem (15) of the descriptor system (1).

The following lemma shows that the form (9) can be used to characterize the existence of a feedback matrix $F$ such that the pencil $(E, A+B F)$ is regular and of index at most one.

Lemma 7. Given a descriptor system of the form (1), there exists a matrix $F$ such that the pencil $(E, A+B F)$ is regular and of index at most one if and only if

$$
n_{3}=\tilde{n}_{3}, \quad E_{23}=0, \quad E_{33}=0 .
$$


Proof. For any $F \in \mathbf{R}^{m \times n}$, denote

$$
\left.W^{T} F V=:\left[\begin{array}{lll}
F_{11} & F_{12} & F_{13} \\
F_{21} & F_{22} & F_{23}
\end{array}\right]\right\} \begin{aligned}
& \tilde{n}_{2} \\
& \} m-\tilde{n}_{2}
\end{aligned}
$$

We have

$$
\begin{aligned}
& {\left[\begin{array}{cc}
Q_{11} & Q_{12} \\
0 & I
\end{array}\right] U(s E-A-B F) V} \\
& (18)=\left[\begin{array}{ccc}
s E_{11}-A_{11}-B_{12} F_{21} & -A_{12}-B_{12} F_{22} & s E_{13}-A_{13}-B_{12} F_{23} \\
-\left(A_{21}+B_{21} F_{11}\right) & -\left(A_{22}+B_{21} F_{12}\right) & s E_{23}-\left(A_{23}+B_{21} F_{13}\right) \\
0 & 0 & s E_{33}-A_{33}
\end{array}\right] .
\end{aligned}
$$

Necessity. Let $F \in \mathbf{R}^{m \times n}$ be such that the pencil $(E, A+B F)$ is regular and of index at most one. Then by the regularity of $(E, A+B F)$ we have

$$
\max _{s \in \mathbf{C}} \operatorname{rank}(s E-A-B F)=n,
$$

which together with (18) yields that

$$
\max _{s \in \mathbf{C}} \operatorname{rank}\left(s E_{33}-A_{33}\right)=\tilde{n}_{3} .
$$

Note that $s E_{33}-A_{33}$ is of full column rank for any $s \in \mathbf{C}$. Thus,

$$
\tilde{n}_{3}=n_{3} \text {. }
$$

Since the pencil $(E, A+B F)$ is regular and of index at most one, $s E_{33}-A_{33}$ is of full column rank for any $s \in \mathbf{C}$, and we have using Lemma 4(iii) and (19) that

$$
\begin{aligned}
\operatorname{rank}(E)= & \operatorname{deg}(\operatorname{det}(s E-A-B F)) \\
= & \operatorname{deg}\left(\operatorname{det}\left(\left[\begin{array}{cc}
s E_{11}-A_{11}-B_{12} F_{21} & -A_{12}-B_{12} F_{22} \\
-\left(A_{21}+B_{21} F_{11}\right) & -\left(A_{22}+B_{21} F_{12}\right)
\end{array}\right]\right)\right. \\
& +\operatorname{deg}\left(\operatorname{det}\left(s E_{33}-A_{33}\right)\right) \\
= & \operatorname{deg}\left(\operatorname{det}\left(\left[\begin{array}{cc}
s E_{11}-A_{11}-B_{12} F_{21} & -A_{12}-B_{12} F_{22} \\
-\left(A_{21}+B_{21} F_{11}\right) & -\left(A_{22}+B_{21} F_{12}\right)
\end{array}\right]\right)\right. \\
\leq & \\
= & \operatorname{rank}\left(E_{11}\right) \\
= & n_{1} .
\end{aligned}
$$

Because $E_{11} \in \mathbf{R}^{n_{1} \times n_{1}}$ is nonsingular, we also have

$$
\operatorname{rank}(E)=\operatorname{rank}\left(E_{11}\right)+\operatorname{rank}\left[\begin{array}{c}
E_{23} \\
E_{33}
\end{array}\right]=n_{1}+\operatorname{rank}\left[\begin{array}{c}
E_{23} \\
E_{33}
\end{array}\right] .
$$

Hence, we obtain

$$
E_{23}=0, \quad E_{33}=0,
$$

which together with (19) give the condition (16).

Sufficiency. Assume condition (16) holds. It follows that $\tilde{n}_{2}=n_{2}$ and $\operatorname{rank}(E)=$ $n_{1}$. Let

$$
F_{12}=-B_{21}^{-1}\left(A_{22}+I\right), \quad F_{11}, F_{13}, F_{21}, F_{22}, \text { and } F_{23} \text { are arbitrary. }
$$

By Lemma 4(ii), we know that $(E, A+B F)$ is regular and of index at most one. 
In Corollary 6, it is shown that systems (1) and (15) have the same set of finite zeros. In the next result it will be shown that the RRDP for descriptor system (1) can be reduced to the RRDP for the linear time-invariant system (15).

THEOREM 8. Given a descriptor system (1), the RRDP for system (1) is solvable if and only if the condition (16) holds and, furthermore,

the RRDP for system (15) is solvable;

i.e., there exist matrices $\mathcal{F}$ and $\mathcal{H}$ with $\mathcal{H}$ nonsingular such that

$\mathcal{T}_{\mathcal{F}, \mathcal{H}}(s)=\left(\left(C_{1}+\left[\begin{array}{ll}0 & C_{2}\end{array}\right] \mathcal{F}\right)\left(s E_{11}-A_{11}-\left[\begin{array}{ll}B_{12} & A_{12}\end{array}\right] \mathcal{F}\right)^{-1}\left[\begin{array}{ll}B_{12} & A_{12}\end{array}\right]+\left[\begin{array}{ll}0 & C_{2}\end{array}\right]\right) \mathcal{H}$ (25)

is nonsingular and diagonal.

Proof. For any $F \in \mathbf{R}^{m \times n}$ and $H \in \mathbf{R}^{m \times m}$, denote $W^{T} F V$ as in (17), and let

$$
\tilde{H}=W^{T} H \text {. }
$$

Clearly, if $H$ is nonsingular, then $\tilde{H}$ is nonsingular. We will first prove "necessity" and then "sufficiency."

Necessity. Let $F \in \mathbf{R}^{m \times n}$ and $H \in \mathbf{R}^{m \times m}$ with $H$ nonsingular be such that the pencil $(E, A+B F)$ is regular, of index at most one, and (4) is true. Then condition (16) of the theorem follows directly from Lemma 7. Note that the condition (16) implies that

$$
\tilde{n}_{2}=n_{2}, \quad \operatorname{rank}(E)=n_{1} .
$$

We have shown in the proof of Lemma 7 that (20) holds, from which it follows that the pencil

$$
\left(\left[\begin{array}{cc}
E_{11} & 0 \\
0 & 0
\end{array}\right],\left[\begin{array}{ll}
A_{11}+B_{12} F_{21} & A_{12}+B_{12} F_{22} \\
A_{21}+B_{21} F_{11} & A_{22}+B_{21} F_{12}
\end{array}\right]\right)
$$

is regular and of index at most one. Hence, by Lemma 4 (ii), we have that $A_{22}+B_{21} F_{12}$ is nonsingular. Now a simple calculation yields that

$$
\begin{aligned}
& C(s E-A-B F)^{-1} B H \\
= & {\left[\begin{array}{lll}
C_{1} & C_{2} & C_{3}
\end{array}\right]\left[\begin{array}{ccc}
s E_{11}-A_{11}-B_{12} F_{21} & -A_{12}-B_{12} F_{22} & s E_{13}-A_{13}-B_{12} F_{23} \\
-\left(A_{21}+B_{21} F_{11}\right) & -\left(A_{22}+B_{21} F_{12}\right) & -\left(A_{23}+B_{21} F_{13}\right) \\
0 & 0 & -A_{33}
\end{array}\right] } \\
& \times\left[\begin{array}{cc}
0 & B_{12} \\
B_{21} & 0 \\
0 & 0
\end{array}\right] \tilde{H} \\
= & {\left[\begin{array}{cc}
C_{1} & C_{2}
\end{array}\right]\left[\begin{array}{cc}
s E_{11}-A_{11}-B_{12} F_{21} & -A_{12}-B_{12} F_{22} \\
-\left(A_{21}+B_{21} F_{11}\right) & -\left(A_{22}+B_{21} F_{12}\right)
\end{array}\right]^{-1}\left[\begin{array}{cc}
0 & B_{12} \\
B_{21} & 0
\end{array}\right] \tilde{H} } \\
= & \mathcal{T}_{\mathcal{F}, \mathcal{H}}(s),
\end{aligned}
$$

where

$$
\begin{aligned}
& \mathcal{F}=\left[\begin{array}{c}
F_{21}-F_{22}\left(A_{22}+B_{21} F_{12}\right)^{-1}\left(A_{21}+B_{21} F_{11}\right) \\
-\left(A_{22}+B_{21} F_{12}\right)^{-1}\left(A_{21}+B_{21} F_{11}\right)
\end{array}\right], \\
& \mathcal{H}=\left[\begin{array}{cc}
-F_{22}\left(A_{22}+B_{21} F_{12}\right)^{-1} & I \\
-\left(A_{22}+B_{21} F_{12}\right)^{-1} & 0
\end{array}\right]\left[\begin{array}{cc}
B_{21} & 0 \\
0 & I
\end{array}\right] \tilde{H} .
\end{aligned}
$$


Since $\tilde{H}, B_{21}$, and $A_{22}+B_{21} F_{12}$ are nonsingular, so is $\mathcal{H}$. Hence, the nonsingularity and diagonality of $C(s E-A-B F)^{-1} B H$ imply that $\mathcal{T}_{\mathcal{F}, \mathcal{H}}(s)$ is nonsingular and diagonal. This is equivalent to the solvability of the RRDP for system (15).

Sufficiency. We will prove the sufficiency constructively. Assume that conditions (16) and (24) hold. Condition (16) implies that $n_{2}=\tilde{n}_{2}$, and so the system (15) is square. From the condition (24) there are matrices $\mathcal{F}$ and $\mathcal{H}$ with $\mathcal{H}$ nonsingular and

$$
\mathcal{F}=\left[\begin{array}{l}
\mathcal{F}_{1} \\
\mathcal{F}_{2}
\end{array}\right] \begin{aligned}
& 3 m-n_{2} \\
& \} n_{2}
\end{aligned}
$$

such that $\mathcal{T}_{\mathcal{F}, \mathcal{H}}(s)$ is nonsingular and diagonal. Let $(F, H)$ be determined by

$$
\left\{\begin{array}{l}
{\left[\begin{array}{cc}
B_{21} & 0 \\
0 & I
\end{array}\right] W^{T} F=\left[\begin{array}{ccc}
\mathcal{F}_{2}-A_{21} & -I-A_{22} & 0 \\
\mathcal{F}_{1} & 0 & 0
\end{array}\right] V^{T},} \\
{\left[\begin{array}{cc}
B_{21} & 0 \\
0 & I
\end{array}\right] W^{T} H=\left[\begin{array}{cc}
0 & I_{n_{2}} \\
I_{m-n_{2}} & 0
\end{array}\right] \mathcal{H},}
\end{array}\right.
$$

partition $F$ as in (17), and define $\tilde{H}$ by (26). We have that $A_{22}+B_{21} F_{12}=-I$ and (29) and (30) hold. By condition (16) and the proof of the sufficiency of Lemma 7, $(E, A+B F)$ is regular and of index at most one. Moreover, (28) yields that $C(s E-A-B F)^{-1} B H$ is nonsingular and diagonal.

In general, $\left[\begin{array}{ll}0 & C_{2}\end{array}\right] \neq 0$, and, consequently, Theorem 1 cannot be extended to system (15). Hence, we reduce the RRDP for system (15) to the one for a system of the form (5) via the following factorization.

THEOREM 9. Given a descriptor system (1) and assuming that the form (9) has been determined, then there exist orthogonal matrices $\mathcal{U}, \mathcal{V}$, and $\mathcal{W}$ and a permutation matrix $\mathcal{P}$ such that

$$
\left[\begin{array}{cc}
\mathcal{U} & 0 \\
0 & \mathcal{P}
\end{array}\right]\left[\begin{array}{cccc}
s E_{11}-A_{11} & \mid & B_{12} & A_{12} \\
---- & - & -- & -- \\
C_{1} & \mid & 0 & C_{2}
\end{array}\right]\left[\begin{array}{cc}
\mathcal{V} & 0 \\
0 & \mathcal{W}
\end{array}\right]
$$

$$
\begin{array}{llll}
\mu_{1} & \mu_{2} & \nu & m+n_{2}-\tilde{n}_{2}-\nu
\end{array}
$$

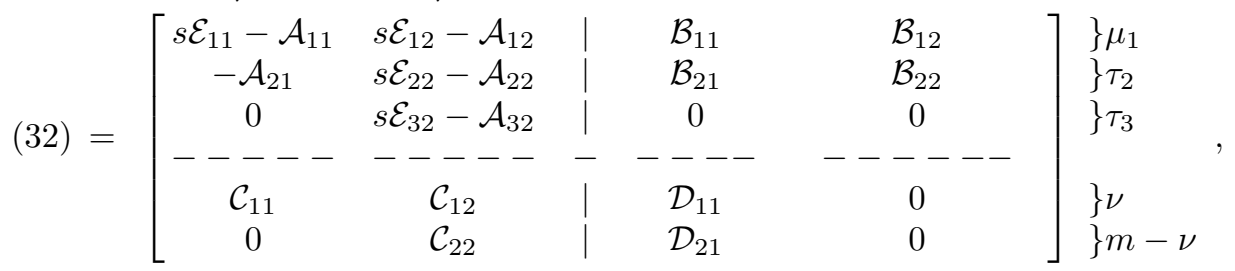

where $\mu_{1}+\mu_{2}=\mu_{1}+\tau_{2}+\tau_{3}=n_{1}, \mathcal{E}_{11}$ and $\mathcal{D}_{11}$ are nonsingular, $\left[\begin{array}{cc}\mathcal{B}_{21} & \mathcal{B}_{22}\end{array}\right]$ is of full row rank, and

$$
\begin{aligned}
\operatorname{rank}\left[\begin{array}{ccc}
s \mathcal{E}_{11}-\mathcal{A}_{11} & \mathcal{B}_{11} & \mathcal{B}_{12} \\
-\mathcal{A}_{21} & \mathcal{B}_{21} & \mathcal{B}_{22}
\end{array}\right] & =\mu_{1}+\tau_{2} \quad \forall s \in \mathbf{C}, \\
\max _{s \in \mathbf{C}} \operatorname{rank}\left[\begin{array}{c}
s \mathcal{E}_{32}-\mathcal{A}_{32} \\
\mathcal{C}_{22}
\end{array}\right] & =\mu_{2} .
\end{aligned}
$$

Proof. The forms (32) are constructed in [23]. 
Let

$\left.\left.\left.\left.\mathcal{V}^{T} \tilde{x}=\left[\begin{array}{l}x_{11} \\ x_{21}\end{array}\right]\right\} \mu_{1}, \quad \mathcal{W}^{T} \tilde{u}=\left[\begin{array}{l}u_{11} \\ u_{21}\end{array}\right]\right\} \begin{array}{l}\} \nu+n_{2}-\tilde{n}_{2}-\nu \\ \} \mu_{2}\end{array}, \quad \mathcal{P} y=\left[\begin{array}{l}y_{1} \\ y_{2}\end{array}\right]\right\}\right\}_{m-\nu}$.

Then system (15) is equivalent to

$$
\left\{\begin{array}{l}
\mathcal{E}_{11} \dot{x}_{11}+\mathcal{E}_{12} \dot{x}_{21}=\mathcal{A}_{11} x_{11}+\mathcal{A}_{12} x_{21}+\mathcal{B}_{11} u_{11}+\mathcal{B}_{12} u_{21} \\
{\left[\begin{array}{l}
\mathcal{E}_{22} \\
\mathcal{E}_{32}
\end{array}\right] \dot{x}_{21}=\left[\begin{array}{c}
\mathcal{A}_{22} \\
\mathcal{A}_{32}
\end{array}\right] x_{21}+\left[\begin{array}{cc}
\mathcal{A}_{21} & \mathcal{B}_{21} \\
0 & 0
\end{array}\right]\left[\begin{array}{c}
x_{11} \\
u_{11}
\end{array}\right]+\left[\begin{array}{c}
\mathcal{B}_{22} \\
0
\end{array}\right] u_{21}} \\
y_{1}=\mathcal{C}_{11} x_{11}+\mathcal{C}_{12} x_{21}+\mathcal{D}_{11} u_{11} \\
y_{2}=\mathcal{C}_{22} x_{21}+\mathcal{D}_{21} u_{11}
\end{array}\right.
$$

Now, the nonsingularity of $E_{11}$ implies that

$$
\left[\begin{array}{l}
\mathcal{E}_{22} \\
\mathcal{E}_{32}
\end{array}\right]
$$

is nonsingular. So, if $\mathcal{B}_{22}$ is nonsingular, we take $u_{11}=0$, and we denote $v_{21}=$ $u_{21}+\mathcal{B}_{22}^{-1} \mathcal{A}_{21} x_{11}$, then the system (36) becomes

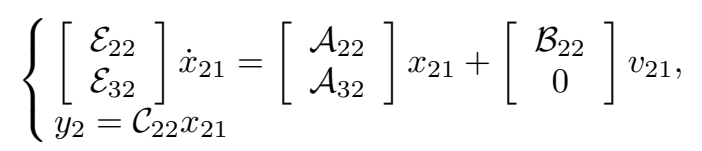

and

$$
\left\{\begin{array}{c}
\mathcal{E}_{11} \dot{x}_{11}=\left(\mathcal{A}_{11}-\mathcal{B}_{12} \mathcal{B}_{22}^{-1} \mathcal{A}_{21}\right) x_{11}+\left(\mathcal{A}_{12}-\mathcal{E}_{12}\left[\begin{array}{c}
\mathcal{E}_{22} \\
\mathcal{E}_{32}
\end{array}\right]^{-1}\left[\begin{array}{c}
\mathcal{A}_{22} \\
\mathcal{A}_{32}
\end{array}\right]\right) x_{21} \\
+\left(\mathcal{B}_{12}-\mathcal{E}_{12}\left[\begin{array}{c}
\mathcal{E}_{22} \\
\mathcal{E}_{32}
\end{array}\right]^{-1}\left[\begin{array}{c}
\mathcal{B}_{22} \\
0
\end{array}\right]\right) v_{21} \\
y_{1}=\mathcal{C}_{11} x_{11}+\mathcal{C}_{12} x_{21}
\end{array}\right.
$$

The main feature of system (37) is that it is a linear time-invariant system of the form (5) because

$$
\left[\begin{array}{l}
\mathcal{E}_{22} \\
\mathcal{E}_{32}
\end{array}\right]
$$

is nonsingular.

Remark 1 . In the descriptions above, we set $u_{11}=0$ to motivate how the system (37) may be deduced from the system (15) by a particular choice of the input. Hence, if the RRDP for the system (15) is solvable, so must the RRDP for the system (37) (i.e., the latter is a necessary condition for the former). However, whether the RRDP for the system (15) is solvable or not should not depend on the choice of the input. Indeed, in Theorem 10, we show that a necessary and sufficient condition for the solvability of the RRDP for the system (15) can be expressed in terms of the solvability of the RRDP for the system (37) independent of the choice of the input, and an examination of the proof of Theorem 10 will reveal that we never make use of $u_{11}=0$ in the proof. To summarize, the descriptions above are just a simple argument intended to introduce the form of the system (37) for easy reference in Theorem 10; 
otherwise, the choice $u_{11}=0$ is not used in the rigorous proof of the equivalence between the RRDPs for the systems (15) and (37).

The following theorem shows that we can reduce the RRDP for system (15) further to the RRDP for system (37).

THEOREM 10. Given a descriptor system (1), suppose the forms (9) and (32) have been determined. Assume that the condition (16) is true. Then the RRDP for system (15) is solvable if and only if

$$
\mathcal{D}_{21}=0, \quad \mathcal{B}_{22} \text { is nonsingular, }
$$

and, furthermore,

$$
\text { the RRDP for system (37) is solvable. }
$$

Proof. Since $n_{3}=\tilde{n}_{3}$ in the form (9), $n_{2}=\tilde{n}_{2}$ and

$$
m+n_{2}-\tilde{n}_{2}-\nu=m-\nu, \quad \mathcal{B}_{12} \in \mathbf{R}^{\mu_{1} \times(m-\nu)}, \quad \mathcal{B}_{22} \in \mathbf{R}^{\tau_{2} \times(m-\nu)} .
$$

Necessity. Assume that $\mathcal{F}$ and $\mathcal{H}$ with $\mathcal{H}$ nonsingular solve the RRDP of system $(15), \mathcal{T}_{\mathcal{F}, \mathcal{H}}(s)$ defined by $(25)$ being diagonal and nonsingular. Let

$$
\left.\left.\left.\mathcal{W}^{T} \mathcal{F} \mathcal{V}=\left[\begin{array}{cc}
\mu_{1} & \mu_{2} \\
\mathcal{F}_{11} & \mathcal{F}_{12} \\
\mathcal{F}_{21} & \mathcal{F}_{22}
\end{array}\right]\right\}\right\}_{m-\nu}, \quad \mathcal{W}^{T} \mathcal{H P}^{T}=\left[\begin{array}{cc}
\nu & m-\nu \\
\mathcal{H}_{11} & \mathcal{H}_{12} \\
\mathcal{H}_{21} & \mathcal{H}_{22}
\end{array}\right]\right\} \nu, \nu
$$

Since $\mathcal{P}$ is a permutation matrix, $\mathcal{P} \mathcal{T}_{\mathcal{F}, \mathcal{H}}(s) \mathcal{P}^{T}$ is also diagonal and nonsingular, or, equivalently,

$$
\begin{aligned}
& {\left[\begin{array}{ll}
\mathcal{T}_{11}(s) & \mathcal{T}_{12}(s) \\
\mathcal{T}_{21}(s) & \mathcal{T}_{22}(s)
\end{array}\right] } \\
= & \left(\left[\begin{array}{ll}
\mathcal{D}_{11} & 0 \\
\mathcal{D}_{21} & 0
\end{array}\right]+\left[\begin{array}{cc}
\mathcal{C}_{11}+\mathcal{D}_{11} \mathcal{F}_{11} & \mathcal{C}_{12}+\mathcal{D}_{11} \mathcal{F}_{12} \\
\mathcal{D}_{21} \mathcal{F}_{11} & \mathcal{C}_{22}+\mathcal{D}_{21} \mathcal{F}_{12}
\end{array}\right]\right. \\
& \left.\times\left(\mathcal{U}\left(s E_{11}-A_{11}-\left[\begin{array}{ll}
B_{12} & A_{12}
\end{array}\right] \mathcal{F}\right) \mathcal{V}\right)^{-1}\left[\begin{array}{cc}
\mathcal{B}_{11} & \mathcal{B}_{12} \\
\mathcal{B}_{21} & \mathcal{B}_{22} \\
0 & 0
\end{array}\right]\right)\left[\begin{array}{ll}
\mathcal{H}_{11} & \mathcal{H}_{12} \\
\mathcal{H}_{21} & \mathcal{H}_{22}
\end{array}\right] \\
= & \mathcal{P} \mathcal{T}_{\mathcal{F}, \mathcal{H}}(s) \mathcal{P}^{T}
\end{aligned}
$$

is diagonal and nonsingular; here

$$
\mathcal{T}_{11}(s) \in \mathbf{R}^{\nu \times \nu}, \mathcal{T}_{12}(s) \in \mathbf{R}^{\nu \times(m-\nu)}, \mathcal{T}_{21}(s) \in \mathbf{R}^{(m-\nu) \times \nu}, \mathcal{T}_{22}(s) \in \mathbf{R}^{(m-\nu) \times(m-\nu)},
$$

so we get

$$
\mathcal{T}_{11}(s) \text { and } \mathcal{T}_{22}(s) \text { are diagonal and nonsingular, } \mathcal{T}_{12}(s)=0, \mathcal{T}_{21}(s)=0 .
$$

Hence, we have

$$
\begin{gathered}
\mathcal{D}_{11} \mathcal{H}_{12}+\left[\begin{array}{cc}
\mathcal{C}_{11}+\mathcal{D}_{11} \mathcal{F}_{11} & \mathcal{C}_{12}+\mathcal{D}_{11} \mathcal{F}_{12}
\end{array}\right]\left(\mathcal{U}\left(s E_{11}-A_{11}-\left[\begin{array}{ll}
B_{12} & A_{12}
\end{array}\right] \mathcal{F}\right) \mathcal{V}\right)^{-1} \\
\times\left[\begin{array}{c}
\mathcal{B}_{11} \mathcal{H}_{12}+\mathcal{B}_{12} \mathcal{H}_{22} \\
\mathcal{B}_{21} \mathcal{H}_{12}+\mathcal{B}_{22} \mathcal{H}_{22} \\
0
\end{array}\right] \quad=\mathcal{T}_{12}(s)=0,
\end{gathered}
$$


694

DELIN CHU AND Y. S. HUNG

$$
\begin{aligned}
& \mathcal{D}_{21} \mathcal{H}_{11}+\left[\begin{array}{cc}
\mathcal{D}_{21} \mathcal{F}_{11} & \mathcal{C}_{22}+\mathcal{D}_{21} \mathcal{F}_{12}
\end{array}\right]\left(\mathcal{U}\left(s E_{11}-A_{11}-\left[\begin{array}{ll}
B_{12} & A_{12}
\end{array}\right] \mathcal{F}\right) \mathcal{V}\right)^{-1} \\
& \times\left[\begin{array}{c}
\mathcal{B}_{11} \mathcal{H}_{11}+\mathcal{B}_{12} \mathcal{H}_{21} \\
\mathcal{B}_{21} \mathcal{H}_{11}+\mathcal{B}_{22} \mathcal{H}_{21} \\
0
\end{array}\right] \\
& =\mathcal{T}_{21}(s)=0
\end{aligned}
$$

Thus, Lemma 2(i) gives that

$$
\mathcal{D}_{11} \mathcal{H}_{12}=0, \quad \mathcal{D}_{21} \mathcal{H}_{11}=0
$$

Since $\mathcal{D}_{11}$ and

$$
\left[\begin{array}{ll}
\mathcal{H}_{11} & \mathcal{H}_{12} \\
\mathcal{H}_{21} & \mathcal{H}_{22}
\end{array}\right]
$$

are nonsingular, we have

$$
\mathcal{H}_{12}=0, \quad \mathcal{D}_{21}=0, \quad \mathcal{H}_{11} \text { and } \mathcal{H}_{22} \text { are nonsingular }
$$

Using (45), (43) becomes

(46) $\left[\begin{array}{ll}0 & \mathcal{C}_{22}\end{array}\right]\left(\mathcal{U}\left(s E_{11}-A_{11}-\left[\begin{array}{ll}B_{12} & A_{12}\end{array}\right] \mathcal{F}\right) \mathcal{V}\right)^{-1}\left[\begin{array}{c}\mathcal{B}_{11}+\mathcal{B}_{12} \mathcal{H}_{21} \mathcal{H}_{11}^{-1} \\ \mathcal{B}_{21}+\mathcal{B}_{22} \mathcal{H}_{21} \mathcal{H}_{11}^{-1} \\ 0\end{array}\right]=0$

By Lemma 2(i) and properties (34) and (46), we get

$$
\begin{aligned}
& n_{1}=\mu_{1}+\mu_{2}=\max _{s \in \mathbf{C}} \operatorname{rank}\left[\begin{array}{r}
\mathcal{U}\left(s E_{11}-A_{11}-\left[\begin{array}{ll}
B_{12} & A_{12}
\end{array}\right] \mathcal{F}\right) \mathcal{V}\left[\begin{array}{c}
\mathcal{B}_{11}+\mathcal{B}_{12} \mathcal{H}_{21} \mathcal{H}_{11}^{-1} \\
\mathcal{B}_{21}+\mathcal{B}_{22} \mathcal{H}_{21} \mathcal{H}_{11}^{-1} \\
0 \\
0
\end{array}\right] \\
0
\end{array}\right] \\
& =\max _{s \in \mathbf{C}} \operatorname{rank}\left[\begin{array}{ccc}
s \mathcal{E}_{11}-\mathcal{A}_{11}-\mathcal{B}_{11} \mathcal{F}_{11}-\mathcal{B}_{12} \mathcal{F}_{21} & s \mathcal{E}_{12}-\mathcal{A}_{12}-\mathcal{B}_{11} \mathcal{F}_{12}-\mathcal{B}_{12} \mathcal{F}_{22} & \mathcal{B}_{11}+\mathcal{B}_{12} \mathcal{H}_{21} \mathcal{H}_{11}^{-1} \\
-\mathcal{A}_{21}-\mathcal{B}_{21} \mathcal{F}_{11}-\mathcal{B}_{22} \mathcal{F}_{21} & s \mathcal{E}_{22}-\mathcal{A}_{22}-\mathcal{B}_{21} \mathcal{F}_{12}-\mathcal{B}_{22} \mathcal{F}_{22} & \mathcal{B}_{21}+\mathcal{B}_{22} \mathcal{H}_{21} \mathcal{H}_{11}^{-1} \\
0 & s \mathcal{E}_{32}-\mathcal{A}_{32} & 0 \\
0 & \mathcal{C}_{22} & 0
\end{array}\right] \\
& =\max _{s \in \mathbf{C}} \operatorname{rank}\left[\begin{array}{cc}
s \mathcal{E}_{11}-\mathcal{A}_{11}-\mathcal{B}_{11} \mathcal{F}_{11}-\mathcal{B}_{12} \mathcal{F}_{21} & \mathcal{B}_{11}+\mathcal{B}_{12} \mathcal{H}_{21} \mathcal{H}_{11}^{-1} \\
-\mathcal{A}_{21}-\mathcal{B}_{21} \mathcal{F}_{11}-\mathcal{B}_{22} \mathcal{F}_{21} & \mathcal{B}_{21}+\mathcal{B}_{22} \mathcal{H}_{21} \mathcal{H}_{11}^{-1}
\end{array}\right]+\mu_{2} \\
& \geq \operatorname{rank}\left(\mathcal{E}_{11}\right)+\operatorname{rank}\left(\mathcal{B}_{21}+\mathcal{B}_{22} \mathcal{H}_{21} \mathcal{H}_{11}^{-1}\right)+\mu_{2} \quad \text { (by Lemma 3(i)) } \\
& =\mu_{1}+\mu_{2}+\operatorname{rank}\left(\mathcal{B}_{21}+\mathcal{B}_{22} \mathcal{H}_{21} \mathcal{H}_{11}^{-1}\right) \text {. }
\end{aligned}
$$

Thus,

$$
\mathcal{B}_{21}+\mathcal{B}_{22} \mathcal{H}_{21} \mathcal{H}_{11}^{-1}=0
$$

which implies that

$$
\operatorname{rank}\left(\mathcal{B}_{22}\right)=\operatorname{rank}\left[\begin{array}{cc}
\mathcal{B}_{21} & \mathcal{B}_{22}
\end{array}\right]
$$


By construction, $\left[\begin{array}{cc}\mathcal{B}_{21} & \mathcal{B}_{22}\end{array}\right]$ is of full row rank $\left(=\tau_{2}\right)$. Hence,

$$
\operatorname{rank}\left(\mathcal{B}_{22}\right)=\tau_{2}
$$

Note that $\mathcal{E}_{11}$ is nonsingular and $\mathcal{B}_{22}$ is of full row rank (see (50)); Lemma 3(ii) yields that

$$
\max _{s \in \mathbf{C}} \operatorname{rank}\left[\begin{array}{cc}
s \mathcal{E}_{11}-\mathcal{A}_{11}-\mathcal{B}_{11} \mathcal{F}_{11}-\mathcal{B}_{12} \mathcal{F}_{21} & \mathcal{B}_{12} \\
-\mathcal{A}_{21}-\mathcal{B}_{21} \mathcal{F}_{11}-\mathcal{B}_{22} \mathcal{F}_{21} & \mathcal{B}_{22}
\end{array}\right]=\mu_{1}+\tau_{2}
$$

Since

$\mathcal{T}_{22}(s)=\left[\begin{array}{ll}0 & \mathcal{C}_{22}\end{array}\right]\left(\mathcal{U}\left(s E_{11}-A_{11}-\left[\begin{array}{ll}B_{12} & A_{12}\end{array}\right] \mathcal{F}\right) \mathcal{V}\right)^{-1}\left[\begin{array}{c}\mathcal{B}_{12} \\ \mathcal{B}_{22} \\ 0\end{array}\right] \mathcal{H}_{22} \in \mathbf{R}^{(m-\nu) \times(m-\nu)}$

is diagonal and nonsingular, and $E_{11} \in \mathbf{R}^{n_{1} \times n_{1}}$, we have using (34), (50), (51), and the nonsingularity of $\mathcal{H}_{22}$ and $\mathcal{E}_{11}$ that

$$
\begin{aligned}
& n_{1}+(m-\nu)=\mu_{1}+\mu_{2}+(m-\nu)
\end{aligned}
$$

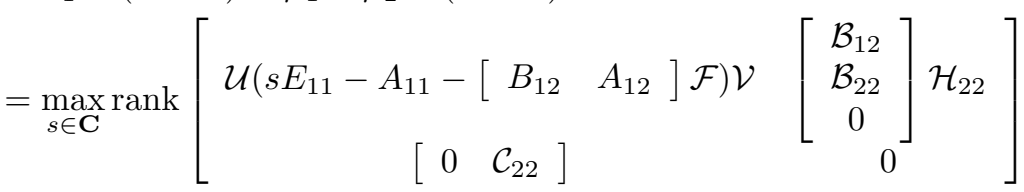

$$
\begin{aligned}
& =\max _{s \in \mathbf{C}} \operatorname{rank}\left[\begin{array}{ccc}
s \mathcal{E}_{11}-\mathcal{A}_{11}-\mathcal{B}_{11} \mathcal{F}_{11}-\mathcal{B}_{12} \mathcal{F}_{21} & s \mathcal{E}_{12}-\mathcal{A}_{12}-\mathcal{B}_{11} \mathcal{F}_{12}-\mathcal{B}_{12} \mathcal{F}_{22} & \mathcal{B}_{12} \mathcal{H}_{22} \\
-\mathcal{A}_{21}-\mathcal{B}_{21} \mathcal{F}_{11}-\mathcal{B}_{22} \mathcal{F}_{21} & s \mathcal{E}_{22}-\mathcal{A}_{22}-\mathcal{B}_{21} \mathcal{F}_{12}-\mathcal{B}_{22} \mathcal{F}_{22} & \mathcal{B}_{22} \mathcal{H}_{22} \\
0 & s \mathcal{E}_{32}-\mathcal{A}_{32} & 0 \\
0 & \mathcal{C}_{22} & 0
\end{array}\right] \\
& =\max _{s \in \mathbf{C}} \operatorname{rank}\left[\begin{array}{cc}
s \mathcal{E}_{11}-\mathcal{A}_{11}-\mathcal{B}_{11} \mathcal{F}_{11}-\mathcal{B}_{12} \mathcal{F}_{21} & \mathcal{B}_{12} \\
-\mathcal{A}_{21}-\mathcal{B}_{21} \mathcal{F}_{11}-\mathcal{B}_{22} \mathcal{F}_{21} & \mathcal{B}_{22}
\end{array}\right]+\mu_{2} \\
& =\left(\mu_{1}+\tau_{2}\right)+\mu_{2} \\
& \text { (by Lemma 3(ii)). }
\end{aligned}
$$

Hence,

$$
\tau_{2}=m-\nu .
$$

Since $\mathcal{B}_{22} \in \mathbf{R}^{\tau_{2} \times(m-\nu)}$ (see (40)), it follows from (50) and (52) that $\mathcal{B}_{22}$ is nonsingular, which together with (45) proves that condition (38) of the theorem holds.

Using the nonsingularity of $\mathcal{B}_{22}$, we have from (48) that

$$
\mathcal{H}_{21} \mathcal{H}_{11}^{-1}=-\mathcal{B}_{22}^{-1} \mathcal{B}_{21}
$$

which gives that

$$
\mathcal{B}_{11}+\mathcal{B}_{12} \mathcal{H}_{21} \mathcal{H}_{11}^{-1}=\mathcal{B}_{11}-\mathcal{B}_{12} \mathcal{B}_{22}^{-1} \mathcal{B}_{21}
$$

From (47), (48), and (54), we obtain

$$
\begin{aligned}
\mu_{1} & =\max _{s \in \mathbf{C}} \operatorname{rank}\left[\begin{array}{cc}
s \mathcal{E}_{11}-\mathcal{A}_{11}-\mathcal{B}_{11} \mathcal{F}_{11}-\mathcal{B}_{12} \mathcal{F}_{21} & \mathcal{B}_{11}-\mathcal{B}_{12} \mathcal{B}_{22}^{-1} \mathcal{B}_{21} \\
-\mathcal{A}_{21}-\mathcal{B}_{21} \mathcal{F}_{11}-\mathcal{B}_{22} \mathcal{F}_{21} & 0
\end{array}\right] \\
= & \max _{s \in \mathbf{C}} \operatorname{rank}\left[\begin{array}{cc}
s \mathcal{E}_{11}-\mathcal{A}_{11}-\mathcal{B}_{11} \mathcal{F}_{11}-\mathcal{B}_{12} \mathcal{F}_{21}-\mathcal{B}_{12} \mathcal{B}_{22}^{-1}\left(-\mathcal{A}_{21}-\mathcal{B}_{21} \mathcal{F}_{11}-\mathcal{B}_{22} \mathcal{F}_{21}\right) & \mathcal{B}_{11}-\mathcal{B}_{12} \mathcal{B}_{22}^{-1} \mathcal{B}_{21} \\
-\mathcal{A}_{21}-\mathcal{B}_{21} \mathcal{F}_{11}-\mathcal{B}_{22} \mathcal{F}_{21} & 0
\end{array}\right] \\
& =\max _{s \in \mathbf{C}} \operatorname{rank}\left[\begin{array}{cc}
s \mathcal{E}_{11}-\mathcal{A}_{11}+\mathcal{B}_{12} \mathcal{B}_{22}^{-1} \mathcal{A}_{21}-\left(\mathcal{B}_{11}-\mathcal{B}_{12} \mathcal{B}_{22}^{-1} \mathcal{B}_{21}\right) \mathcal{F}_{11} & \mathcal{B}_{11}-\mathcal{B}_{12} \mathcal{B}_{22}^{-1} \mathcal{B}_{21} \\
-\mathcal{A}_{21}-\mathcal{B}_{21} \mathcal{F}_{11}-\mathcal{B}_{22} \mathcal{F}_{21} & 0
\end{array}\right] .
\end{aligned}
$$


Consequently, we have

$$
\mu_{1}=\max _{s \in \mathbf{C}} \operatorname{rank}\left[\begin{array}{cc}
s \mathcal{E}_{11}-\mathcal{A}_{11}+\mathcal{B}_{12} \mathcal{B}_{22}^{-1} \mathcal{A}_{21} & \mathcal{B}_{11}-\mathcal{B}_{12} \mathcal{B}_{22}^{-1} \mathcal{B}_{21} \\
-\mathcal{A}_{21}-\mathcal{B}_{21} \mathcal{F}_{11}-\mathcal{B}_{22} \mathcal{F}_{21} & 0
\end{array}\right] .
$$

Because (33) holds and $\mathcal{B}_{22}$ is nonsingular,

$$
\begin{aligned}
\mu_{1}+\tau_{2} & =\operatorname{rank}\left[\begin{array}{ccc}
s \mathcal{E}_{11}-\mathcal{A}_{11} & \mathcal{B}_{11} & \mathcal{B}_{12} \\
-\mathcal{A}_{21} & \mathcal{B}_{21} & \mathcal{B}_{22}
\end{array}\right] \\
& =\operatorname{rank}\left[\begin{array}{ccc}
s \mathcal{E}_{11}-\mathcal{A}_{11}+\mathcal{B}_{12} \mathcal{B}_{22}^{-1} \mathcal{A}_{21} & \mathcal{B}_{11}-\mathcal{B}_{12} \mathcal{B}_{22}^{-1} \mathcal{B}_{21} & 0 \\
-\mathcal{A}_{21} & \mathcal{B}_{21} & \mathcal{B}_{22}
\end{array}\right] \quad \forall s \in \mathbf{C},
\end{aligned}
$$

which gives that

$$
\operatorname{rank}\left[s \mathcal{E}_{11}-\mathcal{A}_{11}+\mathcal{B}_{12} \mathcal{B}_{22}^{-1} \mathcal{A}_{21} \quad \mathcal{B}_{11}-\mathcal{B}_{12} \mathcal{B}_{22}^{-1} \mathcal{B}_{21}\right]=\mu_{1} \quad \forall s \in \mathbf{C} .
$$

Thus, by applying Lemma 2(ii) to (55) we get

$$
\mathcal{A}_{21}+\mathcal{B}_{21} \mathcal{F}_{11}+\mathcal{B}_{22} \mathcal{F}_{21}=0 .
$$

Since $\mathcal{B}_{22}$ is nonsingular, $\mathcal{B}_{21} \mathcal{F}_{12}+\mathcal{B}_{22} \mathcal{F}_{22}=\mathcal{B}_{22} \tilde{\mathcal{F}}_{22}$ with $\tilde{\mathcal{F}}_{22}=\mathcal{B}_{22}^{-1} \mathcal{B}_{21} \mathcal{F}_{12}+\mathcal{F}_{22}$, and

$$
\begin{aligned}
\mathcal{C}_{22} & {\left[\begin{array}{c}
s \mathcal{E}_{22}-\mathcal{A}_{22}-\mathcal{B}_{22} \tilde{\mathcal{F}}_{22} \\
s \mathcal{E}_{32}-\mathcal{A}_{32}
\end{array}\right]^{-1}\left[\begin{array}{c}
\mathcal{B}_{22} \\
0
\end{array}\right] \mathcal{H}_{22} } \\
\quad & {\left[\begin{array}{ll}
0 & \mathcal{C}_{22}
\end{array}\right]\left(\mathcal{U}\left(s E_{11}-A_{11}-\left[\begin{array}{ll}
B_{12} & A_{12}
\end{array}\right] \mathcal{F}\right) \mathcal{V}\right)^{-1}\left[\begin{array}{c}
\mathcal{B}_{12} \\
\mathcal{B}_{22} \\
0
\end{array}\right] \mathcal{H}_{22} }
\end{aligned}
$$

is diagonal and nonsingular. Hence, the condition (39) of the theorem follows.

Sufficiency. We will prove the sufficiency constructively. Assume that conditions (38) and (39) hold. Since $n_{3}=\tilde{n}_{3}$ implies $n_{2}=\tilde{n}_{2}$, the system (37) is square. From the condition (39) there are matrices $\tilde{\mathcal{F}}_{22}$ and $\mathcal{H}_{22}$ such that

$$
\mathcal{T}_{22}(s)=\mathcal{C}_{22}\left[\begin{array}{c}
s \mathcal{E}_{22}-\mathcal{A}_{22}-\mathcal{B}_{22} \tilde{\mathcal{F}}_{22} \\
s \mathcal{E}_{32}-\mathcal{A}_{32}
\end{array}\right]^{-1}\left[\begin{array}{c}
\mathcal{B}_{22} \\
0
\end{array}\right] \mathcal{H}_{22} \text { is diagonal and nonsingular. }
$$

Define $(\mathcal{F}, \mathcal{H})$ by

$$
\left\{\begin{array}{l}
{\left[\begin{array}{cc}
\mathcal{D}_{11} & 0 \\
\mathcal{B}_{21} & \mathcal{B}_{22}
\end{array}\right] \mathcal{W}^{T} \mathcal{H P}^{\mathcal{T}}=\left[\begin{array}{cc}
I & 0 \\
0 & \mathcal{B}_{22} \mathcal{H}_{22}
\end{array}\right],} \\
{\left[\begin{array}{cc}
\mathcal{D}_{11} & 0 \\
\mathcal{B}_{21} & \mathcal{B}_{22}
\end{array}\right] \mathcal{W}^{T} \mathcal{F} \mathcal{V}=\left[\begin{array}{cc}
-\mathcal{C}_{11} & -\mathcal{C}_{12} \\
-\mathcal{A}_{21} & \mathcal{B}_{22} \tilde{\mathcal{F}}_{22}
\end{array}\right] .}
\end{array}\right.
$$

and partition $\mathcal{W}^{T} \mathcal{F V}$ and $\mathcal{W}^{T} \mathcal{H} \mathcal{P}^{T}$ as in (41). A direct calculation yields that

$$
\mathcal{D}_{11} \mathcal{H}_{11}=I, \quad \mathcal{B}_{21} \mathcal{F}_{12}+\mathcal{B}_{22} \mathcal{F}_{22}=\mathcal{B}_{22} \tilde{\mathcal{F}}_{22},
$$

and, furthermore,

$$
\mathcal{T}_{\mathcal{F}, \mathcal{H}}(s)=\mathcal{P}^{T}\left[\begin{array}{cc}
I & 0 \\
0 & \mathcal{T}_{22}(s)
\end{array}\right] \mathcal{P}
$$


is diagonal and nonsingular. Therefore, $\mathcal{F}$ and $\mathcal{H}$ above solve the RRDP for system (15).

By combining Theorems 1,8 , and 10 we obtain the following result, which presents explicit and numerically verifiable necessary and sufficient solvability conditions for the RRDP of system (1).

THEOREM 11. Given a descriptor system (1) and assuming that the forms (9) and (32) have been determined, then the following statements are equivalent:

(i) The RRDP for descriptor system (1) is solvable.

(ii)

$$
\begin{array}{ll}
\text { (a) } n_{3}=\tilde{n}_{3}, & E_{23}=0, \quad E_{33}=0, \\
\text { (b) } \mathcal{D}_{21}=0, & \mathcal{B}_{22} \text { is nonsingular, }
\end{array}
$$

and the RRDP for linear time-invariant system (37) is solvable.

(iii) The conditions in (a) and (b) above hold and

$$
\text { (c) } \mathcal{L} \text { is nonsingular, }
$$

where $\mathcal{L}$ is defined as follows.

Let $c_{i}$ be the ith row of $\mathcal{C}_{22}$. If

$$
c_{i}\left(\left[\begin{array}{l}
\mathcal{E}_{22} \\
\mathcal{E}_{32}
\end{array}\right]^{-1}\left[\begin{array}{l}
\mathcal{A}_{22} \\
\mathcal{A}_{32}
\end{array}\right]\right)^{j}\left(\left[\begin{array}{c}
\mathcal{E}_{22} \\
\mathcal{E}_{32}
\end{array}\right]^{-1}\left[\begin{array}{c}
\mathcal{B}_{22} \\
0
\end{array}\right]\right) \neq 0
$$

for some nonnegative integer $j$; then set

$$
\begin{aligned}
& l_{i}=\min \{j \geq 0: j \text { is integer satisfying } \\
& \left.c_{i}\left(\left[\begin{array}{l}
\mathcal{E}_{22} \\
\mathcal{E}_{32}
\end{array}\right]^{-1}\left[\begin{array}{l}
\mathcal{A}_{22} \\
\mathcal{A}_{32}
\end{array}\right]\right)^{j}\left(\left[\begin{array}{c}
\mathcal{E}_{22} \\
\mathcal{E}_{32}
\end{array}\right]^{-1}\left[\begin{array}{c}
\mathcal{B}_{22} \\
0
\end{array}\right]\right) \neq 0\right\}
\end{aligned}
$$

otherwise, set $l_{i}=\mu_{2}-1$. Define

$$
\mathcal{L}=\left[\begin{array}{c}
c_{1}\left(\left[\begin{array}{c}
\mathcal{E}_{22} \\
\mathcal{E}_{32}
\end{array}\right]^{-1}\left[\begin{array}{l}
\mathcal{A}_{22} \\
\mathcal{A}_{32}
\end{array}\right]\right)^{l_{1}} \\
c_{2}\left(\left[\begin{array}{c}
\mathcal{E}_{22} \\
\mathcal{E}_{32}
\end{array}\right]^{-1}\left[\begin{array}{l}
\mathcal{A}_{22} \\
\mathcal{A}_{32}
\end{array}\right]\right)^{l_{2}} \\
\vdots \\
c_{m-\nu}\left(\left[\begin{array}{c}
\mathcal{E}_{22} \\
\mathcal{E}_{32}
\end{array}\right]^{-1}\left[\begin{array}{c}
\mathcal{A}_{22} \\
\mathcal{A}_{32}
\end{array}\right]\right)^{l_{m-\nu}}
\end{array}\right]\left(\left[\begin{array}{c}
\mathcal{E}_{22} \\
\mathcal{E}_{32}
\end{array}\right]^{-1}\left[\begin{array}{c}
\mathcal{B}_{22} \\
0
\end{array}\right]\right) .
$$

It is well known that it is ill-conditioned to compute the matrix $\mathcal{L}$ in Theorem 11(iii). Hence, Theorem 11(iii) cannot be used for the purpose of numerical computation [18]. Fortunately, the RRDP for system (37), in which

$$
\left[\begin{array}{l}
\mathcal{E}_{22} \\
\mathcal{E}_{32}
\end{array}\right]
$$

is nonsingular, has been reinvestigated, and a numerically reliable algorithm has been developed using orthogonal transformations in $[14,16]$. As a result, Theorem 11(ii), 
the proofs of Theorems 8 and 10, and the work in $[14,16]$ can be used as a basis for devising a numerically reliable algorithm for solving the RRDP for the descriptor system (1) as follows.

Algorithm 1.

Input: Matrices $E, A, B$, and $C$ of the system (1) with $E$ singular.

Output: Solution $(F, H)$ of the RRDP for system (1) if the solution exists.

Step 1. Compute the form (9); check conditions in (a) of Theorem 11(ii). If these conditions are satisfied, go to Step 2; otherwise, conclude that "The RRDP is not solvable" and stop.

Step 2. Compute the form (32); check condition in (b) of Theorem 11(ii). If these conditions are satisfied, go to Step 3; otherwise, conclude that "The RRDP is not solvable" and stop.

Step 3. Verify the solvability conditions of the RRDP for the linear time-invariant system (37) and compute a solution $\left(\tilde{\mathcal{F}}_{22}, \mathcal{H}_{22}\right)$ (if possible) using the algorithm developed in $[16]$.

Step 4. Solve the 4 linear equations in (59) and (31) to get $(F, H)$. Output $(F, H)$ and stop.

In Algorithm 1, Steps 1, 2, and 3 are implemented using only orthogonal transformations, and the equations in Step 4 can be solved by existing reliable methods in MATLAB software. Therefore, Algorithm 1 is numerically reliable.

In the following we present a numerical example to illustrate Algorithm 1. In this example, all calculations were carried out using MATLAB 5.3 on a HP 712/80 workstation with IEEE standard; i.e., the machine accuracy is about $\epsilon \cong 10^{-16}$. For the sake of space limitation, we display only the matrices in systems (1), (15), and (37) and the computed $(F, H)$. But, all other data produced by Algorithm 1 can be obtained from us on request.

Example 1. Given a system of the form (1) with

$$
\begin{aligned}
& {\left[\begin{array}{cccccc}
-2.114533471754 & -1.370853194916 & 1.736011048459 & -0.777424723706 & 0.15552253682 & -2.56440325995 \\
1.981948836367 & 1.02648953419 & -1.105079726788 & 1.060541636692 & 0.15747945272 & 1.83814732774 \\
-1.12769234885 & -0.738768389781 & 0.831143443676 & -0.43067648973 & 0.0746892963 & -1.60807586863
\end{array}\right]}
\end{aligned}
$$

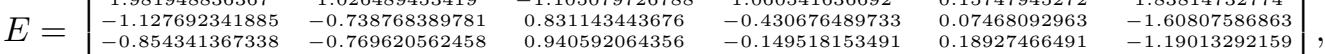

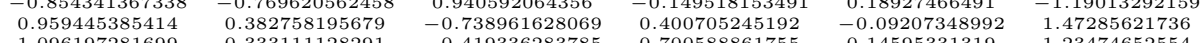

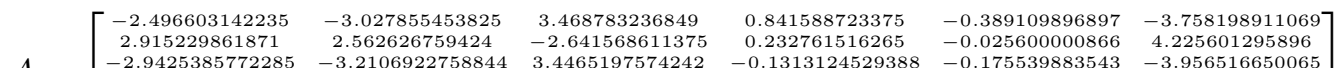

$$
\begin{aligned}
& A=\left[\begin{array}{cccccc}
-2.9425385772285 & -3.2106922758844 & 3.4465197574242 & -0.1313124529388 & -0.175539883543 & -3.956516650065 \\
-2.489894380756 & -2.570286952960 & 1.313484388828 & 0.586156437213 & 0.670989681583 & -3.581315152567 \\
-0.021382994553 & -0.390533814618 & 0.523695683833 & 0.011304370558 & 0.011554521246 & -0.807953060870 \\
1.419644861011 & 0.660544600602 & -0.674155524801 & 0.845044224805 & -0.061255771247 & 1.272523221691
\end{array}\right] \\
& B=\left[\begin{array}{ccc}
-0.2981194205345 & 0.2863134942399 & 0.2343502478597 \\
-0.1599567951551 & -0.0253643526707 & 0.2901408282503 \\
0.2845183665656 & 0.1741030084725 & -0.2934607414939 \\
-0.4542405355796 & -0.2318865773690 & 0.0001128595941 \\
0.1872510988007 & -0.0171206916918 & -0.0015501494030 \\
-0.3507039683173 & 0.0264223889745 & 0.4739000261467
\end{array}\right] \\
& C=\left[\begin{array}{ccccccc}
-0.166889510228 & 0.038161721675 & 0.362202814343 & 0.989416167409 & -0.191104341881 & 0.172703849180 \\
0.010071486000 & 0.008210117004 & 0.005330380954 & 0.012683631440 & 0.010082426509 & -0.026547375104 \\
-1.212588186646 & -0.619627725178 & 0.990749246465 & -0.455865810296 & 0.152486891422 & -1.845755890228
\end{array}\right],
\end{aligned}
$$

$E$ is singular with $\operatorname{rank}(E)=5$.

By performing Step 1 of Algorithm 1 we get the form (9) with

$$
n_{1}=5, n_{2}=\tilde{n}_{2}=1, n_{3}=\tilde{n}_{3}=0, E_{23} \text { and } E_{33} \text { are nonexistent }
$$

$$
E_{11}=\left[\begin{array}{ccccc}
-1.81412863731296 & 0.42421095468505 & -1.05025682473091 & 3.17662461567576 & -1.81554610479108 \\
-2.28707883576749 & 0.58790381967586 & -0.87731476780594 & 3.11620021773695 & -2.16544496029582 \\
-0.48446826993548 & 0.42267233234963 & -0.15485265545682 & 0.41083294329550 & -0.28637255352499 \\
-0.44261627208646 & -0.22062625954174 & -0.18343402237887 & 1.30497574966312 & -1.04899876010130 \\
0.73772319004855 & -0.40897839106130 & 0.02906644815678 & -0.44508380911416 & 0.59700805882825
\end{array}\right]
$$




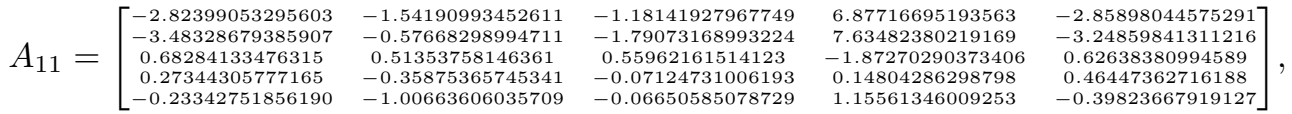

$$
\begin{aligned}
& {\left[B_{12} \mid A_{12}\right]=\left[\begin{array}{cc|c}
-0.29338446075126 & -0.22855442644477 & -0.66970485705469 \\
0.02262609006324 & 0.57657906855771 & -0.24241575506484 \\
-0.03965740026352 & -0.07378004229509 & 0.01290081567965 \\
-0.32758200246128 & -0.43860929198782 & -0.44527719790514 \\
-0.14453716656447 & -0.41240236312705 & -0.25082197786380
\end{array}\right],} \\
& C_{1}=\left[\begin{array}{ccccc}
-0.49764149376588 & 0.26436703986119 & -0.37975128183368 & -0.525589367331624 & -0.339826433711893 \\
-0.00466385565791 & 0.01770707047205 & -0.01384911053026 & -0.00520420774033 & 0.02470573280247 \\
1.07461245340293 & -0.12658398822948 & 0.34423979427567 & -1.76367786178704 & 1.44010323440087
\end{array}\right] \text {, } \\
& C_{2}=\left[\begin{array}{c}
0.59356291253968 \\
0 \\
0
\end{array}\right]
\end{aligned}
$$

So, the condition (16) is true.

Next, we perform Step 2 of Algorithm 1 to get the form (32) with

$$
\begin{aligned}
& \mu_{1}=1, \mu_{2}=4, \tau_{2}=2, \tau_{3}=2, \nu=1, \mathcal{D}_{21}=0, \mathcal{B}_{22} \text { is nonsingular, } \\
{\left[\begin{array}{l}
\mathcal{E}_{22} \\
\mathcal{E}_{32}
\end{array}\right]=} & {\left[\begin{array}{cccc}
0.18497650851300 & 0.21650255217584 & -1.42163757723914 & 0.06052836892962 \\
0.14215088563157 & 0.08522385113982 & -6.06459778219956 & 1.67086227506901 \\
0.00000000000000 & 0.00753734517815 & -0.05947922313868 & 0.05399731930089 \\
-0.13125083366301 & -0.20761568732362 & -1.17186177742605 & -0.20101487098613
\end{array}\right], } \\
{\left[\begin{array}{c}
\mathcal{A}_{22} \\
\mathcal{A}_{32}
\end{array}\right]=} & {\left[\begin{array}{cccc}
1.98285434904403 & 1.32236482538160 & -6.66396698837071 & 2.82024406998733 \\
2.52659314741932 & 1.78106640040819 & -8.93198215464291 & 3.05066915354195 \\
-0.00000000000000 & 0.01488524911679 & 0.06423512937203 & -0.04810327759609 \\
-0.25920285056388 & -0.41001322791008 & 0.12457919258231 & 0.11493631853526
\end{array}\right], } \\
{\left[\begin{array}{c}
\mathcal{B}_{22} \\
0
\end{array}\right]=} & {\left[\begin{array}{cccc}
-0.17545104068710 & -0.03097433768157 \\
-0.13911242684627 & 0.26719805271403 \\
0 & 0 & 0
\end{array}\right], } \\
\mathcal{C}_{22}= & {\left[\begin{array}{cccc}
0 & 0 & 0 & 0.03412545630891 \\
0 & 0 & -2.35658869878193 & 0.95930344402099
\end{array}\right] . }
\end{aligned}
$$

Hence, the condition (38) holds.

Then, by performing Step 3 of Algorithm 1 , we get a solution $\left(\tilde{\mathcal{F}}_{22}, \mathcal{H}_{22}\right)$ to the RRDP for system (37):

$\tilde{\mathcal{F}}_{22}=\left[\begin{array}{cccc}9.802293309432168 & 5.646580746820156 & -35.08609913450191 & 14.41371812628298 \\ -3.301832971710871 & -3.096022324474101 & 10.20273393604259 & -1.043725946131937\end{array}\right]$

and

$$
\mathcal{H}_{22}=\left[\begin{array}{cc}
0.9996702178578495 & -0.7886455744849721 \\
-0.02 .567986619979914 & 0.6148480770444584
\end{array}\right] .
$$

Finally, by solving the four linear equations in Step 4 of Algorithm 1, we get

$$
H=\left[\begin{array}{ccc}
-0.17686381243266 & -0.46126177248064 & -0.11010692440574 \\
-5.21439030830574 & -0.29532442684054 & 0.55048173448973 \\
1.10055215419784 & 0.83667261229188 & -0.82755442430760
\end{array}\right] \text {, }
$$


$F=\left[\begin{array}{cccccc}2.2179774813366 & 3.178794801661 & -4.860781467075 & 0.199813884087 & 0.281334640565 & 8.543338100611 \\ 6.031618125212 & 7.960378436260 & -7.806500773764 & 4.155869542985 & 0.747155047430 & 9.384264975229 \\ -16.361782614150 & -16.151965525174 & 14.586848000379 & -0.397781332210 & 0.589084350377 & -24.14475367080\end{array}\right]$

Now we verify that the above pair $(F, H)$ is a solution of the RRDP for system (1). By computing the SVD of $E$ using MATLAB code svd.m we obtain orthogonal matrices $W, \mathcal{W} \in \mathbf{R}^{6 \times 6}$ such that $(W E \mathcal{W}, W(A+B F) \mathcal{W}, W(B H), C \mathcal{W})$ is of the following form:

$$
\begin{aligned}
& \begin{array}{llll}
5 & 1 & 5 & 1
\end{array} \\
& \left.\left.W E \mathcal{W}=\left[\begin{array}{cc}
\Theta_{11} & 0 \\
0 & 0
\end{array}\right]\right\} 5, \quad W(A+B F) \mathcal{W}=\left[\begin{array}{ll}
\Phi_{11} & \Phi_{12} \\
\Phi_{21} & \Phi_{22}
\end{array}\right]\right\} 1, \\
& W B H=\left[\begin{array}{l}
\Psi_{1} \\
\Psi_{2}
\end{array}\right] \begin{array}{l}
35 \\
\} 1
\end{array}, \quad C \mathcal{W}=\left[\begin{array}{cc}
5 & 1 \\
\Upsilon_{1} & \Upsilon_{2}
\end{array}\right],
\end{aligned}
$$

where $\Theta_{11}$ is nonsingular and $\Phi_{22}=-1.00000000000004 \neq 0$. Thus, the pencil $(E, A+B F)$ is regular and of index at most one. Furthermore, a simple calculation by using MATLAB code

$C(s E-A-B F)^{-1} B H$

$=\operatorname{tf}\left(\operatorname{ss}\left(\Theta_{11}^{-1}\left(\Phi_{11}-\Phi_{12} \Phi_{22}^{-1} \Phi_{21}\right), \Theta_{11}^{-1}\left(\Psi_{1}-\Phi_{12} \Phi_{22}^{-1} \Psi_{2}\right),\left(\Upsilon_{1}-\Upsilon_{2} \Phi_{22}^{-1} \Phi_{21}\right),-\Upsilon_{2} \Phi_{22}^{-1} \Psi_{2}\right)\right)$

yields that

$$
C(s E-A-B F)^{-1} B H=\left[\begin{array}{lll}
T_{11}(s) & T_{12}(s) & T_{13}(s) \\
T_{21}(s) & T_{22}(s) & T_{23}(s) \\
T_{31}(s) & T_{32}(s) & T_{33}(s)
\end{array}\right]
$$

with

$$
\begin{aligned}
& {\left[\begin{array}{l}
T_{11}(s) \\
T_{21}(s) \\
T_{31}(s)
\end{array}\right]=\left[\begin{array}{c}
\frac{s^{5}-5.466 s^{4}+9.888 s^{3}-5.912 s^{2}-4.865 \times 10^{-14} s-2.039 \times 10^{-28}}{s^{5}-5.466 s^{4}+9.888 s^{3}-5.912 s^{2}-3.147 \times 10^{-14} s-7.126 \times 10^{-28}} \\
\frac{s^{3}-3.950 s^{2}+3.90 s+1.732 \times 10^{-14}}{s^{5}-5.466 s^{4}+9.888 s^{3}-5.912 s^{2}-3.147 \times 10^{-14} s-7.126 \times 10^{-28}} \times 1.292 \times 10^{-15} \\
-\frac{s^{3}-3.949 s^{2}+3.900 s+2.252 \times 10^{-14}}{s^{5}-5.466 s^{4}+9.888 s^{3}-5.912 s^{2}-3.147 \times 10^{-14} s-7.126 \times 10^{-28}} \times 3.444 \times 10^{-14}
\end{array}\right],} \\
& {\left[\begin{array}{l}
T_{12}(s) \\
T_{22}(s) \\
T_{32}(s)
\end{array}\right]=\left[\begin{array}{c}
\frac{s^{5}+1.811 s^{4}-16.65 s^{3}+25.82 s^{2}-15.20 s-7.119 \times 10^{-14}}{s^{5}-5.466 s^{4}+9.888 s^{3}-5.912 s^{2}-3.147 \times 10^{-14} s-7.126 \times 10^{-28}} \times 4.613 \times 10^{-16} \\
\frac{0.005079 s^{4}-0.02776 s^{3}+0.05022 s^{2}-0.03003 s-2.034 \times 10^{-15}}{s^{5}-5.466 s^{4}+9.888 s^{3}-5.912 s^{2}-3.147 \times 10^{-14} s-7.126 \times 10^{-28}} \\
\frac{s^{3}-3.896 s^{2}+5.276 s-2.929}{s^{5}-5.466 s^{4}+9.888 s^{3}-5.912 s^{2}-3.147 \times 10^{-14} s-7.126 \times 10^{-28}} \times 1.958 \times 10^{-14}
\end{array}\right],} \\
& {\left[\begin{array}{l}
T_{13}(s) \\
T_{23}(s) \\
T_{33}(s)
\end{array}\right]=\left[\begin{array}{c}
\frac{s^{5}-8.802 s^{4}-6.047 s^{3}+13.74 s^{2}+3.245 \times 10^{-15} s-2.693 \times 10^{-30}}{s^{5}-5.466 s^{4}+9.888 s^{3}-5.912 s^{2}-3.147 \times 10^{-14} s-7.126 \times 10^{-28}} \times 1.895 \times 10^{-16} \\
\frac{s^{2}-1.841 s-0.2652}{s^{5}-5.466 s^{4}+9.888 s^{3}-5.912 s^{2}-3.147 \times 10^{-14} s-7.126 \times 10^{-28}} 3.659 \times 10^{-15} \\
\frac{0.07654 s^{4}-0.4184 s^{3}+0.7568 s^{2}-0.4526 s+2.889 \times 10^{-14}}{s^{5}-5.466 s^{4}+9.888 s^{3}-5.912 s^{2}-3.147 \times 10^{-14} s-7.126 \times 10^{-28}}
\end{array}\right] .}
\end{aligned}
$$

Although the off-diagonal elements of $C(s E-A-B F)^{-1} B H$ are not exactly zero, the numerator coefficients of all off-diagonal terms are of an order of magnitude $\mathbf{O}\left(10^{-14}\right)$ that are attributed to numerical rounding error. Thus, $C(s E-A-B F)^{-1} B H$ is for practical purposes diagonal and nonsingular. This can also be demonstrated by 
a step response analysis: it has been found that the response to steps or sinusoidal inputs applied on each separate control channel of the closed-loop system effectively gives terms on the off-diagonal parts which are about $\mathbf{O}\left(10^{-14}\right)$. Hence, the above pair $(F, H)$ is a solution of the RRDP for system (1).

Remark 2. We can also verify that $C(s E-A-B F)^{-1} B H$ in Example 1 is diagonal as follows.

Let $(B H)_{i}$ denote the $i$ th column of $B H(i=1,2,3)$. We obtain orthogonal matrices $W_{i}$ and $\mathcal{W}_{i}(i=1,2,3)$ by computing the controllable staircase forms [20] of the pairs $\left(s E-A-B F,(B H)_{i}\right)(i=1,2,3)$ such that $\left(W_{i}(s E-A-B F) \mathcal{W}_{i}, W_{i}(B H)_{i}, C \mathcal{W}_{i}\right)$ $(i=1,2,3)$ are of the following forms:

$$
\begin{aligned}
& \left\{\begin{array}{c}
\left.W_{1}(s E-A-B F) \mathcal{W}_{1}=\left[\begin{array}{cc}
s \Theta_{11}^{(1)}-\Phi_{11}^{(1)} & s \Theta_{12}^{(1)}-\Phi_{12}^{(1)} \\
0 & s \Theta_{22}^{(1)}-\Phi_{22}^{(1)}
\end{array}\right]\right\} 2, \\
4 \\
2 \\
\left.\left.W_{1}(B H)_{1}=\left[\begin{array}{c}
\Psi_{1}^{(1)} \\
0
\end{array}\right]\right\} 2, \quad C \mathcal{W}_{1}=\left[\begin{array}{ll}
\Upsilon_{11}^{(1)} & \Upsilon_{12}^{(1)} \\
\Upsilon_{21}^{(1)} & \Upsilon_{22}^{(1)} \\
\Upsilon_{31}^{(1)} & \Upsilon_{32}^{(1)}
\end{array}\right]\right\} \begin{array}{l}
\} 1 \\
\} 1
\end{array},
\end{array}\right.
\end{aligned}
$$

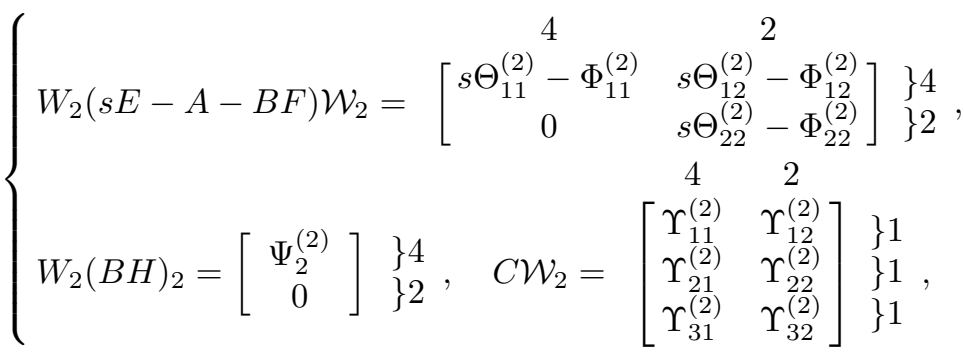

and

$$
\left\{\begin{array}{c}
4 \\
\left.W_{3}(s E-A-B F) \mathcal{W}_{3}=\left[\begin{array}{cc}
s \Theta_{11}^{(3)}-\Phi_{11}^{(3)} & s \Theta_{12}^{(3)}-\Phi_{12}^{(3)} \\
0 & s \Theta_{22}^{(3)}-\Phi_{22}^{(3)}
\end{array}\right]\right\} 2 \\
4 \\
4
\end{array},\right.
$$

where

$$
\begin{aligned}
& \left\|\left[\begin{array}{l}
\Upsilon_{21}^{(1)} \\
\Upsilon_{31}^{(1)}
\end{array}\right]\right\|_{2} /\|C\|_{2}=\mathbf{O}\left(10^{-15}\right), \quad\left\|\left[\begin{array}{c}
\Upsilon_{11}^{(2)} \\
\Upsilon_{31}^{(2)}
\end{array}\right]\right\|_{2} /\|C\|_{2}=\mathbf{O}\left(10^{-15}\right) \\
& \left\|\left[\begin{array}{l}
\Upsilon_{11}^{(3)} \\
\Upsilon_{21}^{(3)}
\end{array}\right]\right\|_{2} /\|C\|_{2}=\mathbf{O}\left(10^{-15}\right)
\end{aligned}
$$

and

$$
\left\|\Upsilon_{11}^{(1)}\right\| /\|C\|=\mathbf{O}(1), \quad\left\|\Upsilon_{21}^{(2)}\right\| /\|C\|=\mathbf{O}(1), \quad\left\|\Upsilon_{31}^{(3)}\right\| /\|C\|=\mathbf{O}(1) .
$$

Therefore, $C(s E-A-B F)^{-1} B H$ is diagonal. 
4. Conclusions. We have presented necessary and sufficient conditions for the solvability of the RRDP for descriptor systems. A numerical procedure, which is implementable and reliable, has been provided to verify these solvability conditions and compute the solution matrices.

Acknowledgment. The authors are grateful to the anonymous referees and Professor B. Kågström for their invaluable comments and suggestions.

\section{REFERENCES}

[1] C. A. Smith, Automated Continuous Process Control, Wiley, New York, 2001.

[2] Q. G. Wang, Decoupling Control, Springer-Verlag, Berlin, 2003.

[3] C.-T. Chen, Linear System Theory and Design, Holt, Rinehart and Winston, New York, 1984.

[4] J. F. Lafay, J. Descusse, and M. Malabre, Solution to Morgan's problem, IEEE Trans. Automat. Control, 33 (1988), pp. 732-739.

[5] J. C. Martinez Garcia And M. Malabre, The simultaneous disturbance rejection and regular row by row decoupling: A geometric approach, IEEE Trans. Automat. Control, 40 (1995), pp. 365-369.

[6] C. Commault, J. M. Dion, and J. Montoya, Simultaneous decoupling and disturbance rejection: A structural approach state space system, Internat. J. Control, 59 (1994), pp. 13251344.

[7] L. DAI, Singular Control Systems, Lecture Notes in Control and Inform. Sci., Vol. 118, SpringerVerlag, Berlin, 1989.

[8] J. W. Demmel AND B. KÅGSTRÖM, The generalized Schur decomposition of an arbitrary pencil $A-\lambda B:$ Robust software with error bounds and applications. Part I: Theory and algorithms, ACM Trans. Math. Software, 19 (1993), pp. 160-174.

[9] E. Fabian And W. M. Wonham, Decoupling and disturbance rejection, IEEE Trans. Automat. Control, 20 (1975), pp. 399-401.

[10] P. L. Falb and W. A. Wolovich, Decoupling in the design and synthesis of multivariable control systems, IEEE Trans. Automat. Control, 12 (1967), pp. 651-659.

[11] M. Christodoulou, Decoupling in the design and synthesis of singular systems, Automatica, 22 (1986), pp. 245-249.

[12] D. Chu and V. Mehrmann, Disturbance decoupling for descriptor systems by state feedback, SIAM J. Control Optim., 38 (2000), pp. 1830-1858.

[13] D. Chu and V. Mehrmann, Disturbance decoupling for linear time-invariant systems: A matrix pencil approach, IEEE Trans. Automat. Control, 46 (2001), pp. 802-808.

[14] D. Chu AND R. TAN, Numerically reliable computing for row by row decoupling problem with stability, SIAM J. Matrix Anal. Appl., 23 (2002), pp. 1143-1170.

[15] D. Chu AND R. TAN, Solvability conditions and parameterization of all solutions for triangular decoupling problem, SIAM J. Matrix Anal. Appl., 23 (2002), pp. 1171-1182.

[16] D. Chu And R. Tan, Numerical Computation for Row by Row Decoupling Problem, Technical report, National University, Singapore, 2000.

[17] A. Bunse-Gerstner, V. Mehrmann, And N. K. Nichols, Regularization of descriptor systems by derivative and proportional state feedback, SIAM J. Matrix Anal. Appl., 13 (1992), pp. $46-67$.

[18] P. H. Petkov, N. D. Christov, and M. M. Konstantinov, Computational Methods in Linear Control Systems, Prentice-Hall, Hertfordshire, UK, 1991.

[19] P. N. Paraskevopulos And F. N. Koumboulis, The decoupling of generalized state-space systems via state feedback, IEEE Trans. Automat. Control, 37 (1992), pp. 148-152.

[20] P. VAn Dooren, The generalized eigenstructure problem in linear system theory, IEEE Trans. Automat. Control, 6 (1981), pp. 111-129.

[21] W. M. Wonham, Linear Multivariable Control: A Geometric Approach, 2nd ed., SpringerVerlag, New York, 1985.

[22] R. Byers, T. Geerts, and V. Mehrmann, Descriptor systems without controllability at infinity, SIAM J. Control Optim., 35 (1997), pp. 462-479.

[23] D. Chu And Y.S. Hung, Row by Row Decoupling Problem for Descriptor Systems, Technical report, National University, Singapore, 2000. 